\title{
The Precedence Effect in Sound Localization
}

\author{
Andrew D. Brown, ${ }^{1}$ G. Christopher Stecker, ${ }^{2}$ and Daniel J. Tollin ${ }^{1}$ \\ ${ }^{1}$ Department of Physiology and Biophysics, University of Colorado School of Medicine, Aurora, CO 80045, USA \\ ${ }^{2}$ Department of Hearing and Speech Sciences, Vanderbilt University Medical Center, Nashville, TN 37232, USA
}

Received: 24 January 2014; Accepted: 13 October 2014; Online publication: 6 December 2014

\begin{abstract}
In ordinary listening environments, acoustic signals reaching the ears directly from real sound sources are followed after a few milliseconds by early reflections arriving from nearby surfaces. Early reflections are spectrotemporally similar to their source signals but commonly carry spatial acoustic cues unrelated to the source location. Humans and many other animals, including nonmammalian and even invertebrate animals, are nonetheless able to effectively localize sound sources in such environments, even in the absence of disambiguating visual cues. Robust source localization despite concurrent or nearly concurrent spurious spatial acoustic information is commonly attributed to an assortment of perceptual phenomena collectively termed "the precedence effect," characterizing the perceptual dominance of spatial information carried by the first-arriving signal. Here, we highlight recent progress and changes in the understanding of the precedence effect and related phenomena.
\end{abstract}

Keywords: precedence effect, echo suppression, sound localization

\section{INTRODUCTION}

Sound source localization is remarkably unaffected by the drastic transformations that source signals undergo in many environments as a result of reflections and reverberation (e.g., Fig. 1). A seminal paper published over six decades ago by Wallach et al. (1949) posited

Correspondence to: Andrew D. Brown - Department of Physiology and Biophysics - University of Colorado School of Medicine. Aurora, CO 80045, USA. Telephone: +1 (303) 724-8960; email: andrew.d.brown@ucdenver.edu that listeners localize sound sources in everyday environments by responding to the acoustic spatial cues carried by the first-arriving sound-that traveling the path directly from the source to the ears-rather than the spurious cues carried by reflected sound arriving milliseconds later from myriad directions unrelated to the source. Wallach et al. (1949) termed this phenomenon the precedence effect in sound localization. Similar phenomena, termed the "Law of the First Wavefront" and the "Haas effect," were described by Cremer (1948) and Haas (1949, 1951) in close chronological proximity to the report of Wallach et al. (1949). Still, earlier observations of a similar nature were reported by Snow (1936) in a patent application and by Fay (1936) and Hall (1936) in conference abstracts. The "precedence effect" label coined by Wallach et al. (1949) has persisted, and it is the one we adopt in this review (for a historical discussion of the precedence effect, including its discovery and nomenclature, see Gardner 1968).

Several hundred studies of the precedence effect and related phenomena since Wallach et al. (1949) have contributed a great deal to our understanding of factors influencing sound localization in everyday (nonanechoic) environments and to our understanding of physiological mechanisms subserving localization across environments. Excellent summaries of earlier studies can be found in the reviews of Zurek (1987), Blauert (1997), and Litovsky et al. (1999). The present review is concerned primarily with studies published during the past $\sim 15$ years, i.e., since the seminal review by Litovsky et al. (1999).

The review is comprised of three sections that consider in turn (1) human psychophysical and noninvasive physiological studies, (2) comparative (animal) psychophysical and physiological studies, and (3) computational models proposed to account for a variety of precedence effect data. We conclude 


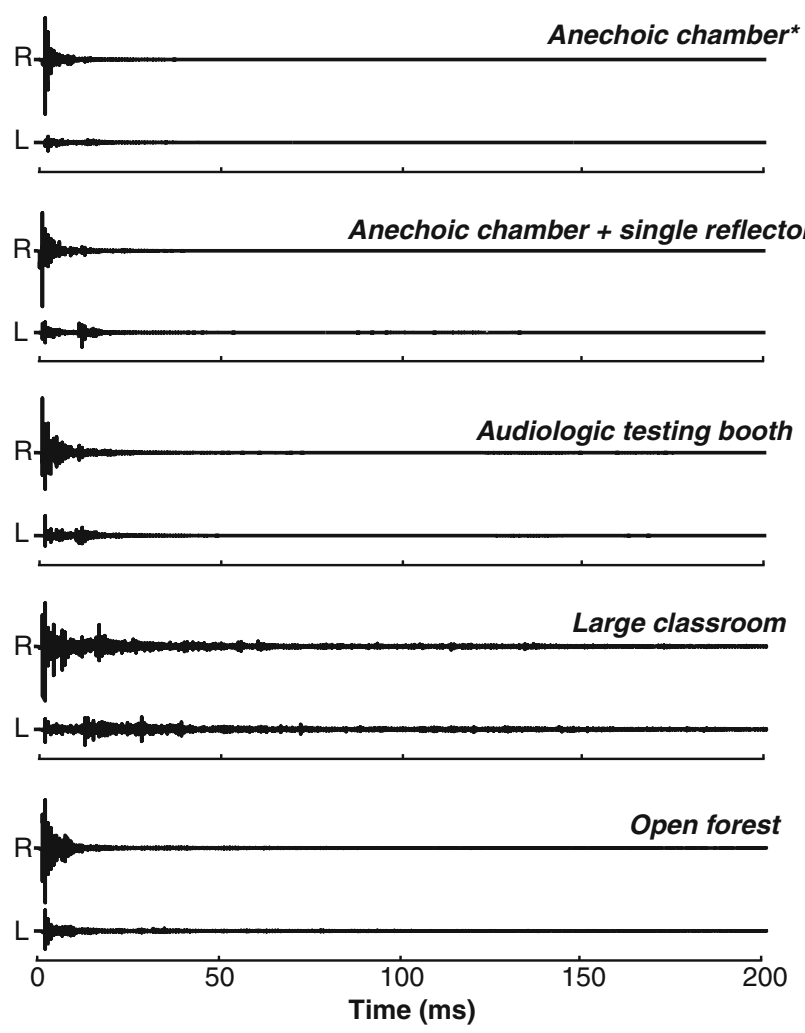

FIG. 1. Binaural recordings of an acoustic impulse in a variety of environments. All recordings were made using a spring-loaded "clapper" as the source, positioned $2 \mathrm{~m}$ directly to the right of a binaural manikin fitted with in-the-canal microphones. The number of discrete reflections and reverberation times produced by nonanechoic environments are widely variable. The "single reflector" recording is the nearest real approximation of the synthetic leadlag transient stimulus used in a majority of studies discussed in this review. The "open forest" recording, made in a deciduous forest in late autumn, illustrates the lack of high-amplitude reflections produced in many natural environments. Within the anechoic chamber, the manikin was situated at the center of a speaker hoop, which produced very slight reflections visible in the left channel particularly (asterisk).

each section with a summary of the major insights offered by the material covered. In a final section, we synthesize major insights of the foregoing sections with particular attention to extant gaps in understanding and remaining challenges in basic and translational research.

\section{HUMAN PSYCHOPHYSICAL STUDIES}

Wallach et al. (1949) demonstrated the precedence effect (PE) using a simple but clever paradigm that has been employed in dozens of investigations since (see also Haas 1949, 1951). In this paradigm, intended to simulate a source signal and single reflection, a human subject is seated equidistant from two loudspeakers in a sound-treated room or anechoic cham- ber and asked to judge features of signals presented through each. In the simplest case, a brief signal such as a click, noise burst, or tone pip is presented through one speaker, followed after some delay by an identical signal presented from the opposite speaker (Fig. 2A). Provided the delay between the first signal (the direct sound, or "lead") and the second signal (the simulated reflection, or "lag") is sufficiently brief, listeners tend to (1) perceive one signal rather than the two that were actually presented, termed fusion, (2) localize the fused signal at or near the location cued by the first of the two signals, termed localization dominance, and (3) lack sensitivity to features of the second signal, most especially its location, termed discrimination suppression (Wallach et al. 1949; Freyman et al. 1991; nomenclature proposed by Litovsky et al. 1999). At longer delays, two discrete signals are perceived near (though not necessarily at) the veridical locations of the lead and lag. At very brief delays of $<1 \mathrm{~ms}$ and especially $<0.5$ $\mathrm{ms}$, listeners tend to perceive a fused image intermediate to the lead and lag, a phenomenon known as "summing localization" (cf. Warncke 1941) that will not be considered extensively in the present review. Some paradigms employ headphones rather than loudspeakers for signal presentation (e.g., Wallach et al. 1949; Zurek 1980), imposing interaural time differences (ITDs), interaural level differences (ILDs), or combinations thereof (e.g., by filtering the signal to each ear with head-related transfer functions (HRTFs) to create a virtual auditory space (VAS) environment (Fig. 2B)).

In either the loudspeaker or headphone version of the "two-source" lead-lag paradigm, subjects are typically instructed to report either the number of sounds perceived, or to localize, lateralize, or discriminate the location or directionality of either the lead or the lag signal. The stimulus variable of primary interest is most often the "lead-lag delay"-the time between the onsets of the lead and lag signals—and performance is usually reported as a function of leadlag delay. The echo threshold is the briefest lead-lag delay at which subjects report perceiving "two sounds" or are able to accurately identify or discriminate the lag location on some criterion proportion of trials (e.g., on $50 \%$ or on $75 \%$ of trials). Performance may also be reported simply in terms of localization accuracy for the lead or lag across lead-lag delay, without explicit reference to a threshold of this type. Differences in stimulus parameters (temporal, spectral, and spatial), tasks employed (including task instructions), and echo threshold definitions (including threshold criteria enforced) have given rise to a wide range of echo thresholds reported in the literature, ranging from 2 to $100 \mathrm{~ms}$ or more (Table 1; see also Litovsky et al. 1999). Substantial variation can 
A Loudspeaker presentation (typically symmetric)

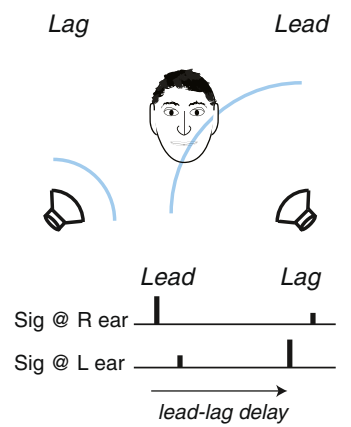

B Headphone presentation

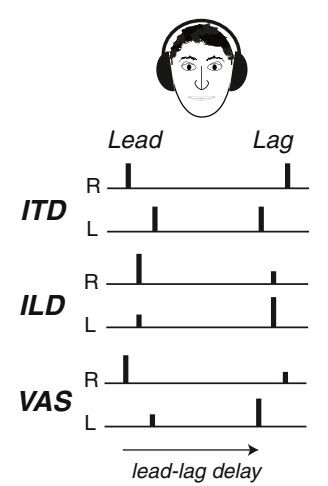

FIG. 2. Common stimulus paradigms for study of the precedence effect. A Free-field stimuli are presented from loudspeakers, most typically positioned symmetrically about the subject at a distance of 1-2 m. The "lead" speaker (right) emits a signal (e.g., a broadband impulse), which is followed after a brief delay by an identical signal from the "lag" speaker (left), typically construed as a simulated "echo." The delay between the lead and lag signals, the "lead-lag delay," is the parameter of primary interest in most studies: Although the lead and lag signals carry two separate sets of spatial acoustic cues, dependent on source azimuth and elevation, the complete stimulus is usually perceived as a single event at one location at brief lead-lag delays. B Stimuli presented over headphones are often used to simulate aspects of free-field lead-lag presentation. Interaural time difference (ITD) stimuli, the most common variant, feature pairs of equal-intensity stimuli presented through each earphone with slight asynchrony $(<1 \mathrm{~ms})$ to produce intracranial (inside-the-head) lateralization of the lead and/or lag. Interaural level difference (ILD) stimuli feature paired stimuli in each channel with intensive asymmetries (but no asynchrony) to produce lateralization of the lead and/or lag. Virtual auditory space (VAS) stimuli, created by filtering stimuli with the head-related transfer functions for the desired lead and lag virtual locations, feature both ITD and ILD cues as well as spectral shape cues in their natural combinations.

also arise from individual subject factors including age and hearing status. Below, we review a variety of such factors studied in recent investigations, and their influences on psychophysical measures of the PE.

\section{Effects of the Task Employed}

Fusion Versus Localization Versus Discrimination-Based Paradigms. The nomenclature proposed by Litovsky et al. (1999) delineates three categories of psychophysical paradigms used to study the PE that may ultimately target different facets of the effect. Toward an improved understanding of the interrelationships among these paradigms, Litovsky and Shinn-Cunningham (2001) employed all three in the same group of subjects. Across experiments, stimuli consisted of 1-ms duration noise bursts presented in lead-lag pairs over headphones with varied lead and lag ITDs. Performance was assessed as a function of lead-lag delay, which was varied systematically. In a fusion experiment, listeners were instructed to report whether they perceived "one fused auditory event or two sounds." In a discrimination suppression experiment, ITD discrimination thresholds were measured adaptively for the lag and separately for the lead. In a final localization dominance experiment, listeners were required to adjust the ITD of a separately presented "pointer" stimulus to match the perceived intracranial position of either the lead or the lag, tested in separate runs. Whereas fusion echo thresholds were 4-7 ms for four of the six subjects, evidence of localization dominance and discrimination suppression persisted to lead-lag delays $>10$ ms, reliably exceeding the fusion echo threshold. The authors interpreted these data to suggest that temporal (fusion) and spatial (localization/discrimination) aspects of the PE may be attributable to different mechanisms.

Other recent studies have also assessed the temporal extent of fusion versus other PE measures. Litovsky and Godar (2010) and Donovan et al. (2012) measured lead and lag localization and fusion echo thresholds using single lead-lag pairs of noise bursts and, concordant with the results of Litovsky and Shinn-Cunningham (2001), found that fusion echo thresholds were significantly lower than the temporal limit of significant spatial lead-lag interactions (e.g., localization dominance). Bianchi et al. (2013) reported similar data for clicks. In contrast, Seeber and Hafter (2011), who tested fusion and both lead and lag localization across a number of conditions using VAS stimuli, concluded that fusion and localization aspects of the PE appeared to follow a similar time course: With increasing lead-lag delay, fusion decreased and the extent of laterality perceived for the lag also gradually increased. This complementary trend is evident when fusion data are plotted as continuous functions (rather than discrete "threshold" values) along with lead/lag lateralization data on the same lead-lag delay axes (see Fig. 3).

Under some conditions, fusion, localization dominance, and discrimination suppression measures of precedence appear to diverge substantially. In a study of "buildup" effects (detailed later in this section; Fig. 4; see also Litovsky et al. 1999), Yang and Grantham (1997) compared echo thresholds measured via subjective fusion versus discrimination suppression tasks in the free field. The authors reported that elevation of the echo threshold by stimulus repetition (i.e., buildup) was several times greater for subjective fusion than for discrimination suppression. Similarly, Brown and Stecker (2013) reported greater changes in fusion than in localization dominance following repetition of headphone ITD and ILD stimuli. These studies suggest that spatial 


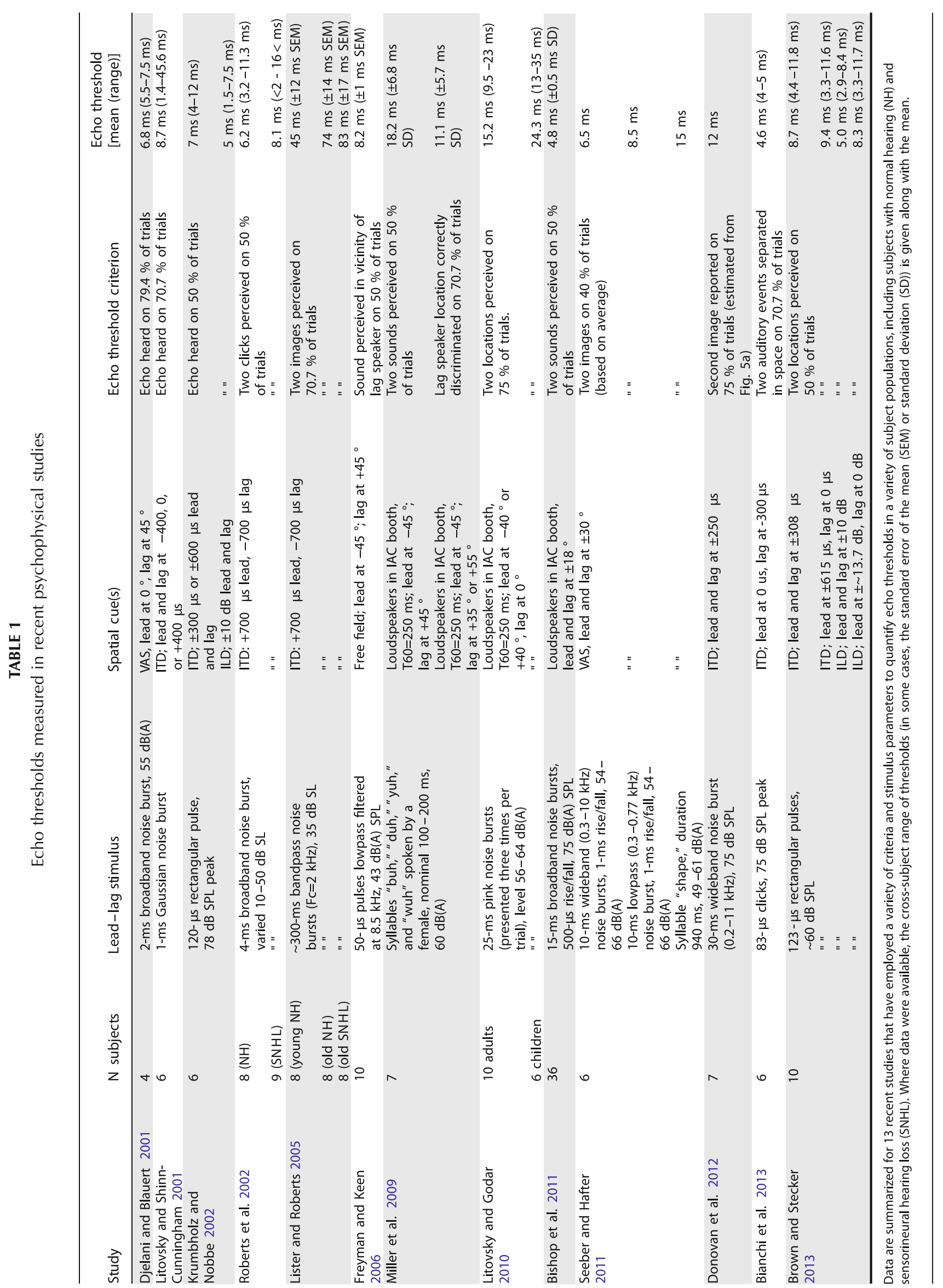


aspects of the PE may be relatively more static across different stimulus configurations, while fusion may be more dynamic.

Insights from Studies of Onset Dominance and Binaural Adaptation. One goal of the present review is to highlight data from psychophysical studies that have employed precedence-like stimuli or paradigms but that may not have been directly motivated by the PE and are not regularly discussed within the PE literature. Specifically, the well-studied effects of onset dominance (e.g., Saberi and Perrot 1995; Freyman et al. 1997; Stecker and Hafter 2002; Stecker and Brown 2010) and binaural adaptation (e.g., Hafter and Dye 1983; Hafter et al. 1983, 1988; Goupell et al. 2009) demonstrate spatiotemporal fusion and localization dominance phenomena quantitatively similar to PEs for paired lead-lag stimuli.

Onset Dominance. Studies of onset dominance typically employ a sequence ("train") of several temporally discrete, spectrally identical or similar stimuli, such as brief impulses or noise carrying different spatial cues in onset (lead) and post-onset (lag) portions of the signal. As in studies of the paired-source PE, stimuli have been presented (1) dichotically, i.e., carrying ITD (e.g., Saberi 1996; Freyman et al. 1997) or ILD (e.g., Stecker and Brown 2010), (2) in a VAS environment (Macpherson and Wagner 2008) or (3) in the free field (Stecker and Hafter 2002, 2009). In each case, subjects are asked to make judgments about the perceived location of the presented stimulus, e.g., via left/right discrimination, via scaled lateralization/pointer adjustment, or via overt pointing at the perceived source location. Consistent with studies of the paired-source PE, listeners' spatial judgments are generally dominated by cues of the onset (lead) when the delay between successive impulses or bursts in the train is briefer than approximately $5 \mathrm{~ms}$ (but see Stecker and Hafter 2009). Saberi (1996) explicitly derived estimates of perceptual "weights" for varied ITD carried by trains of 2-16 discrete pulses. At brief inter-pulse intervals (1.8 and $3 \mathrm{~ms}$ ), consistent with earlier PE studies which had derived analogous weights for the lead and lag (e.g., Shinn-Cunningham et al. 1993), Saberi (1996) demonstrated high weights for the first pulse in a train (the lead), given by discrimination of the fused stimulus consistently in the direction (left or right) of the first pulse ITD, and uniformly low weights for post-onset pulses. At 12-ms inter-pulse interval, weights were similar for onset and post-onset

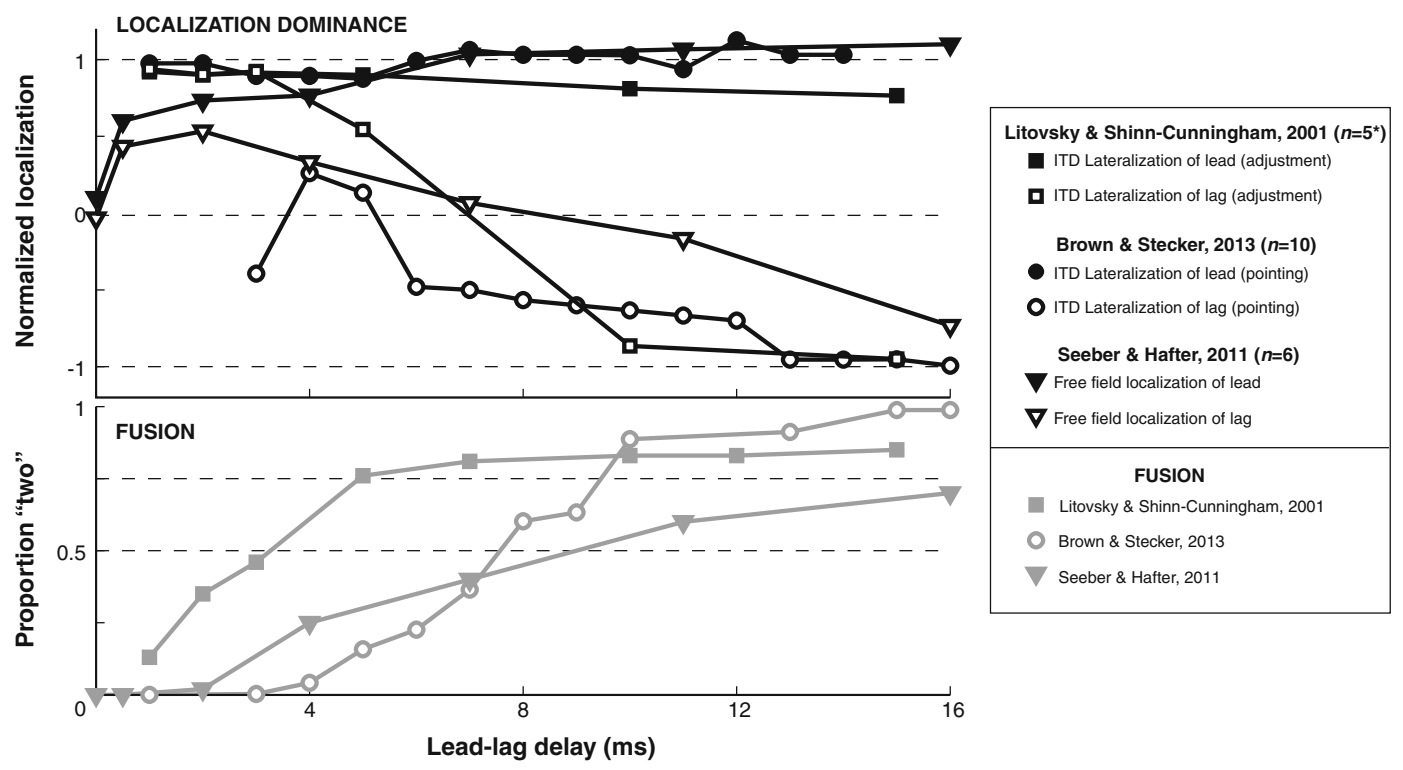

FIG. 3. Comparison of spatial and nonspatial measures of the precedence effect. Mean data are replotted from three studies that assessed as a function of lead-lag delay, in the same groups of subjects, both (1) localization (in VAS) or lateralization of lead and lag signals and (2) subjective fusion (i.e., perception of a "second image," presumed to reflect discrete perception of the lag). Stimuli were 1-ms broadband noise bursts (Litovsky and Shinn-Cunningham 2001), 123- $\mu$ s monophasic pulses (Brown and Stecker 2013), or 10-ms broadband noise bursts (1-ms rise/fall, Seeber and Hafter 2011). Localization data (upper panel) are normalized to the veridical location (localization), target cue (adjustment), or response laterality expected for a "lead-alone" $(+1)$ or "lag-alone" $(-1)$ stimulus (pointing). While localization of the lead was minimally affected by the presence of the lag at any lead-lag delay (beyond the $<1$-ms window of summing localization), localization of the lag was affected by the presence of the lead for lead-lag delays of $10 \mathrm{~ms}$ and beyond. The proportion of trials on which two images were reported (lower panel) increased largely in parallel with increasing lag localization, although the fusion "echo threshold" (lead-lag delay at which each trace crosses the 0.5 or 0.75 (dashed line)) generally occurred before the influence of the lead on lag localization dissipated. Note that these data are for "baseline" (single lead, single lag) stimuli only (see text). Outlying data from a sixth subject in the study of Litovsky and Shinn-Cunningham were omitted (asterisk). 
pulses, consistent with sensitivity to both "leading" and "lagging" information (i.e., weak precedence).

Binaural Adaptation. Studies of binaural adaptation, beginning with an extensive series of experiments by Hafter and colleagues (Hafter and Dye 1983; Hafter et al. 1983, 1988, 1990; Hafter and Buell 1990; Hafter 1997; see also studies by Trahiotis, Bernstein, and colleagues, e.g., Bernstein and Trahiotis 2002), employ stimuli similar to those employed in studies of onset dominance but are different in that the cues of the onset and post-onset portions of the signal are identical (cf. Litovsky and Shinn-Cunningham 2001). Rather than assessing localization or lateralization given by the onset versus post-onset signals, studies of binaural adaptation assess the improvement in discrimination of a static cue as a function of stimulus duration. For example, consistent with studies of the $\mathrm{PE}$, studies of binaural adaptation using pulse train stimuli have generally demonstrated that listeners are rather insensitive to post-onset spatial information when the inter-pulse interval is $\leq 5 \mathrm{~ms}$, evidenced by lack of improvement in detection performance with increasing impulse number (i.e., a failure to integrate information over time for improved sensitivity). At the short inter-pulse interval of $2 \mathrm{~ms}$, for example, listeners' ITD detection thresholds are almost as good for a single pulse (onset only) as for an onset followed by 31 post-onset pulses carrying identical information (Hafter and Dye 1983). When the inter-pulse interval is increased to a larger value, e.g., $14 \mathrm{~ms}$, detection thresholds improve significantly with increasing pulse number, indicating a recovery of sensitivity to postonset information or, in other terms, suggestive that the PE mechanism(s) that limit(s) access to post-onset binaural information is no longer operative. Tollin and Henning (1998) demonstrated similar effects using a traditional two-pulse stimulus and specifically suggested that binaural adaptation and PEs may be produced by the same mechanism(s). We note, as an aside, that studies of binaural adaptation and onset dominance have not to our knowledge formally assessed subjective fusion. Rather, impulse trains produce robustly fused percepts (sounding like a "buzz" or "hum"), even at long inter-pulse intervals for which it can be demonstrated that listeners are sensitive to the spatial cues of each pulse (Stecker and Hafter 2002)—another example of the divergence of fusion versus spatial measures in precedence-like paradigms.

\section{Effects of the Stimulus Spectrum}

Effects of Signal Bandwidth on Localization Dominance. Localization is generally less accurate for narrowband than for broadband sources (e.g., Stevens and Newman 1936), but localization of narrowband sources is particularly poor in reverberant environments (Franssen 1960; Rakerd and Hartmann 1985). Braasch et al. (2003) and Dizon and Colburn (2006) investigated the effects of signal bandwidth on listeners' lateralization of lead-lag noise stimuli. Bandwidth was varied systematically in each study, from 100 to $800 \mathrm{~Hz}$ (Braasch et al. 2003) or from 33 to $1,500 \mathrm{~Hz}$ (Dizon and Colburn 2006). Whereas the broadest bandwidth signals produced robust localization dominance (i.e., lateralization consistently in the direction of the lead) for lead-lag delays up to $\sim 10 \mathrm{~ms}$, consistent with many earlier studies employing broadband transient stimuli (e.g., Shinn-Cunningham et al. 1993), progressively narrower-band noises produced progressively weaker localization dominance. At the narrowest tested bandwidths in both studies, localization dominance was essentially absent, and lateralization strongly favored the lag at some lead-lag delays. The authors explained this result in terms of peripheral filtering effects, i.e., lead-lag interactions prior to and during auditory transduction giving rise to delay-dependent binaural cues in each auditory filter (cf. Tollin and Henning 1999)—a notion detailed in the "Modeling" section of this review.

Effects of Signal Frequency. Human sensitivity to ITD is maximal for signals in the $500-1,000-\mathrm{Hz}$ region of the spectrum (Zwislocki and Feldman 1956; Brughera et al. 2013), and ITD information carried by spectral components in this region appears to supersede localization cues (both ITD and ILD) carried by other components, suggestive of an ITD(or interaural phase)-based "spectral dominance region" in binaural hearing (cf. Bilsen and Raatgever 1973; Gaskell 1983; Heller and Trahiotis 1996). Tollin and Henning (1999) directly assessed the degree to which such a "dominance region" might apply to the PE by presenting listeners with transient "lead-lag" stimuli and "lead-lag-lag" stimuli (simulating a source followed by two echoes, see also Ebata et al. 1968 and Goupell et al. 2012) in a headphone ITD lateralization task. Across a variety of conditions, the authors demonstrated that listeners' lateralization responses were dominated by the effective ITD produced by the composite lead-lag signal in the $\sim 750-\mathrm{Hz}$ region of the spectrum, which at very brief lead-lag delays could spuriously favor the lag, even if the cross-frequency ITD favored the lead (cf. Dizon and Colburn 2006).

Dizon and Colburn (2006) also studied effects of signal center frequency on lateralization responses. Keeping octave-bandwidth constant, localization dominance was shown to become progressively weaker for bandpass noises with $500-\mathrm{Hz}, 1-\mathrm{kHz}$, and $2-\mathrm{kHz}$ center frequencies. The data of Dizon and Colburn (2006) (and those of Tollin and Henning 1999) are qualita- 
tively consistent with earlier data reported by ShinnCunningham et al. (1995), Blauert and Divenyi (1988), and Blauert and Cobben (1978), which also suggested a dominant role for low-frequency information in localization dominance. Whether lowfrequency binaural information is of special importance in the PE specifically or whether its salience in precedence paradigms is merely a manifestation of the dominant role of low-frequency information in spatial hearing in general (Wightman and Kistler 1992; Heller and Trahiotis 1996) is unclear, though we have found no data to contradict the more parsimonious latter account.

\section{Effects of Temporal Parameters}

Effects of Stimulus Duration and Lead-Lag Temporal Overlap. The transient, temporally nonoverlapping stimuli employed in a majority of PE investigations simulate a very limited class of natural sounds. Many natural sounds are of extended duration and give rise to reflections that overlap with the source signal and persist for tens or hundreds of milliseconds after the source signal offset. While a number of studies have employed overlapping stimuli of extended duration to study PEs with more naturalistic (non-transient) stimuli (e.g., Wallach et al. 1949; Zurek 1980), few studies have attempted to contrast the perceptual effects of non-overlapping segments ("lead only" and "lag only") with the lead-lag delay associated with the overlapping segment. Donovan et al. (2012) recently employed a novel stimulus paradigm in which relatively long-duration lead and lag noise bursts were presented dichotically (via imposed ITD) with varying degrees of temporal overlap. In general, data indicated that for a given lead-lag delay, longer duration "lead alone" segments tended to produce a more strongly lateralized image in agreement with the lead ITD and slightly greater lead-lag fusion, while a longer duration "lag alone" stimulus gave weaker lead-lag fusion and stronger lateralization of the lag image. These effects were observed even at very brief lead-lag delays. For example, a 6-ms lead burst that overlapped for $3 \mathrm{~ms}$ with a $27-\mathrm{ms}$ lag burst produced the perception of two images, one on the side of the lead, and one on the side of lag, despite the fact that the lead-lag delay was only $3 \mathrm{~ms}$. A complication in the interpretation of these data arises from the fact that a lagging stimulus of greater duration than its leading stimulus necessarily contains a segment that is novel (i.e., not in the lead), even if spectrally similar. The authors noted that there was little difference observed between this circumstance (longer-duration lag than lead) and the circumstance where the lead and lag were equal durations. However, a discrete reflection carrying a consistent cue for several or tens of milliseconds without contamination from additional reflections carrying conflicting cues is unlikely to occur in a natural setting. Use of this paradigm is considered further in the "Comparative Studies" section of the review (see also Nelson and Takahashi 2008, 2010).

Effects of temporal overlap aside, it has generally been observed that longer-duration stimuli produce a more robust $\mathrm{PE}$ than shorter-duration stimuli. For example, Miller et al. (2009) and Seeber and Hafter (2011) demonstrated longer fusion echo thresholds, greater localization dominance, and stronger lag discrimination suppression with single-syllable speech stimuli (up to 940-ms duration) than for shorterduration stimuli (e.g., 10-ms noise bursts, Seeber and Hafter 2011). Donovan et al. (2012) presented listeners with lead and lag noises of 30 or $200 \mathrm{~ms}$ that were gated on synchronously with a fixed 3-ms lead-lag delay. Localization dominance was shown to be significantly greater for the $200 \mathrm{~ms}$ than for the 30-ms stimulus. Finally, Hafter et al. (2001) assessed both fusion and localization dominance using synthetic piano tones 500 $\mathrm{ms}$ in duration. For equal-amplitude lead and lag tones, echo thresholds for both procedures fell in the range of $7-8 \mathrm{~ms}$; when lagging tones were realistically attenuated, echo thresholds were extended to $15-26 \mathrm{~ms}$, similar to earlier measures obtained with longer-duration speech and music stimuli (e.g., Wallach et al. 1949; Haas 1949, 1951).

Effects of Stimulus Repetition. In addition to effects of duration within single lead-lag pairs, precedence phenomena can be affected by repetition of multiple lead-lag stimuli over a period of several seconds or more. Clifton and colleagues extensively studied the effects of stimulus repetition on fusion (Clifton 1987; Clifton and Freyman 1989; Freyman et al. 1991) and discrimination suppression measures of the $\mathrm{PE}$ (Freyman et al. 1991; Clifton et al. 1994; McCall et al. 1998). Two key phenomena were observed in these and related studies (e.g., Grantham 1996; Yang and Grantham 1997; see Fig. 4): (1) In a phenomenon known as buildup, echo thresholds measured via subjective fusion could increase from $5-10 \mathrm{~ms}$ for a single stimulus presentation to $15-30 \mathrm{~ms}$ or more following several stimulus repetitions; smaller but significant increases in thresholds could also be measured in discrimination suppression tasks (e.g., Yang and Grantham 1997; Djelani and Blauert 2001; see Fig. 4B). (2) In a phenomenon known as "breakdown," repetition-enhanced lead-lag fusion or lag discrimination suppression could be "broken down" to baseline levels following changes to certain features of the lead-lag stimulus. Effective changes included sudden switching of the lead and lag speaker locations (e.g., Clifton and Freyman 1989), a sudden adjustment of the lead-lag delay (Clifton et al. 1994), 
A

A Baseline

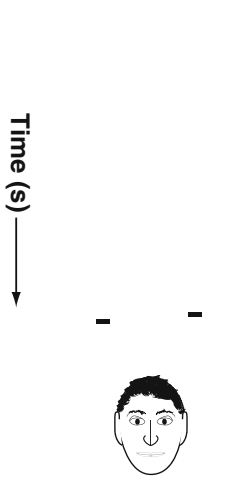

B

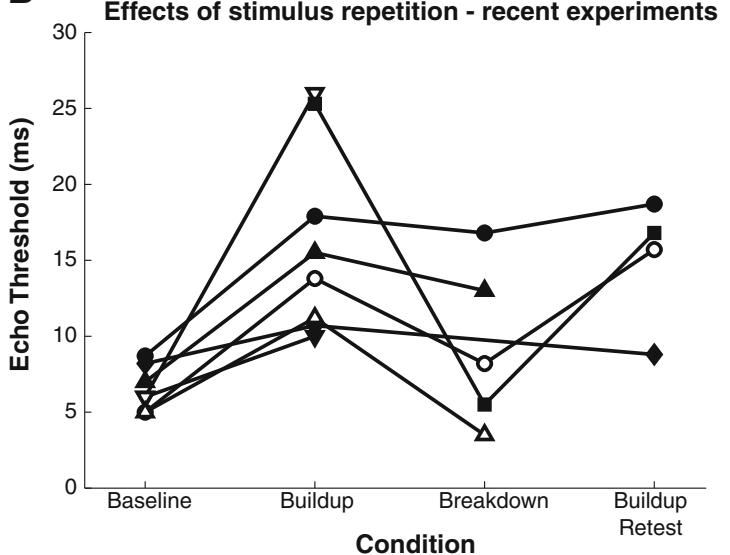

Buildup Breakdown

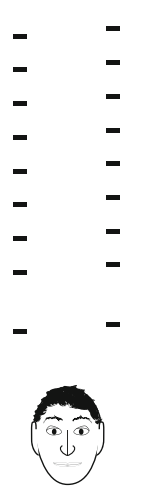

Buildup

Retest

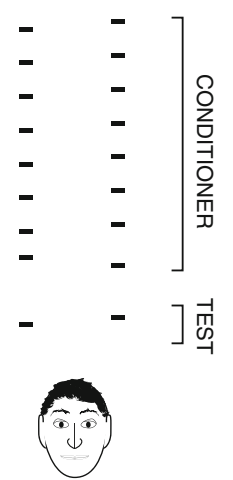

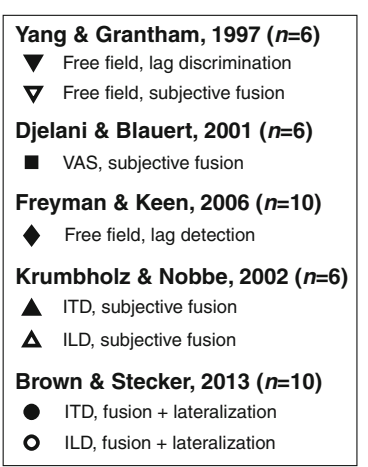

FIG. 4. Effects of repeated stimulus presentation on psychophysical measures of the precedence effect. A Four different stimulus configurations are illustrated. Time is illustrated schematically in the vertical dimension. Baseline stimuli, the configuration used in most studies of the precedence effect, consist of a single lead and lag pair. In this illustration, the lead carries spatial cues favoring the right ear (depicted as a mirror image), and the lag carries spatial cues favoring the left ear, imposed via one of the methods illustrated in Fig. 2. Buildup stimuli consist of a series of lead-lag pairs-a "conditioner" train-carrying spatial cues identical to the test pair, which is presented after a brief pause. Breakdown stimuli (of the type illustrated) consist of a conditioner train carrying spatial cues opposite those of the test stimulus, such that the test stimulus features a "switch" in lead and lag locations. Buildup Retest stimuli are identical to buildup stimuli, with the exception of an intervening lead-lag pair at the end of the conditioner train that carries spatial cues opposite the preceding conditioner pair and the proceeding test pair. B Mean "echo threshold" data are replotted from several experiments that have assessed precedence effects for two or more of

or a sudden shift in the spectrum of the lag (e.g., McCall et al. 1998). In each of these cases, echo thresholds measured for the changed stimulus approximated the echo threshold measured for a single lead-lag stimulus presentation, a result interpreted as a resetting of the echo threshold in response to the sudden change in the lead-lag stimulus. Data from such investigations, in conjunction with the finding of a left-right asymmetry in the buildup effect (see Grantham 1996), were subsequently taken to suggest the stimulus configurations illustrated in $\mathbf{A}$. In all experiments, stimuli were brief broadband noise bursts or impulses. In general, stimulus repetition elevates echo thresholds, but several key findings are that: (1) Response paradigms that require explicit discrimination (Yang and Grantham 1997) of the lag location or detection of an image in the vicinity a visible lag loudspeaker (Freyman and Keen 2006) suggest lesser effects of buildup than subjective paradigms, which generally only require subjects to indicate whether one or two images were perceived. (2) Stimuli that are lateralized by virtue of ILD exclusively (Krumbholz and Nobbe 2002; Brown and Stecker 2013) tend to produce lower echo thresholds than freefield or ITD stimuli in particular. (3) Breakdown of the echo threshold, evidenced by a reduction of the echo threshold relative to the Buildup condition, tends to occur only when ILD cues are present; however, (4) for all cue types, echo thresholds generally remain elevated when the primary conditioner stimulus is presented as the test (e.g., following a "breakdown" stimulus in the Buildup Retest condition).

a role for cognition in the PE (see Litovsky et al. 1999). This view holds that listeners rapidly develop "expectations" about the behavior of sound in a given listening environment, leading to enhanced suppression of the reflected sound (e.g., Clifton et al. 1994; Sanders et al. 2011).

A novel alternative perspective on buildup and breakdown effects was offered by Djelani and Blauert (2001). In their study, listeners were presented with lead-lag stimuli in a VAS environment comprising four different conditions: 
(1) baseline, a single lead-lag stimulus presentation, (2) buildup, a sequence of several identical lead-lag stimuli, (3) breakdown, a sequence of identical lead-lag stimuli followed by an aberrant (spatially switched lead and lag) stimulus, and (4) buildup retest, a breakdown stimulus followed by an additional repetition of the original buildup stimulus (Fig. 4A). Across conditions, the listener's task on each trial was to report the number of sounds perceived for the final stimulus in the sequence (a subjective fusion task). The authors adjusted the lead-lag delay adaptively across trials to measure fusion echo thresholds for each condition. Consistent with earlier studies, echo thresholds in the buildup condition were found to be significantly (twofold to threefold) longer than those in the baseline condition, while thresholds in the breakdown condition were comparable to baseline thresholds. Buildup retest thresholds, in contrast, were comparable to buildup thresholds. That is, despite presentation of the breakdown stimulus, buildup for the repeated stimulus was clearly maintained. The authors took this finding as evidence against a "resetting" of a global echo threshold; rather, by their interpretation, breakdown stimuli simply elicit a baseline echo threshold for a novel stimulus. More recent studies by Freyman and Keen (2006), Keen and Freyman (2009), and Brown and Stecker (2013) have supported this view, demonstrating that buildup may be established for multiple concurrent stimuli and perhaps even in a manner that is specific to individual spatial cues (Krumbholz and Nobbe 2002; Brown and Stecker 2013). Data from recent studies of buildup and breakdown effects are summarized in Figure 4B.

Effects of Spatial Cues. A majority of studies concerned with the PE, including a majority of those discussed in the preceding sections, have employed either free-field or headphone ITD stimuli for measurements of fusion echo thresholds or to assess relative sensitivity to spatial attributes of lead and lag stimuli. A minority of studies have employed headphone ILD or "monaural" spectral shape cues. Therefore, until recently, relatively little was understood about the differential contributions of individual spatial acoustic cues to precedence phenomena.

The Precedence Effect Is More Robust for Stimuli Lateralized by ITD than ILD. Several studies have quantified different aspects of the PE and related phenomena using headphone stimuli lateralized by either ITD or ILD in the same subjects. Zurek (1980), the first to do so, measured lag discrimination performance using binaurally uncorrelated noises with imbedded segments of correlated noise carrying nonzero ITDs or ILDs. At very brief lead-lag delays, ITD discrimination thresholds could not be measured, whereas even at the shortest lead-lag delays,
ILD thresholds were in the range of 10-15 $\mathrm{dB}$. Krumbholz and Nobbe (2002) systematically investigated PEs for ITD and ILD in a subjective lead-lag fusion paradigm. Using pairs of broadband lead-lag clicks presented over headphones that were lateralized to approximately the same intracranial position via either ITD or ILD (directly assessed via a cue-matching task), the authors measured echo thresholds in baseline, buildup, and breakdown conditions. Across all three stimulus types, fusion echo thresholds were shown to be greater for stimuli lateralized by ITD than for stimuli lateralized by ILD. Most strikingly, there was no significant breakdown effect with a "switching" of lead and lag ITD, while switching of lead and lag ILD yielded near-baseline ILD-based echo thresholds. Brown and Stecker (2013) found similar trends in both fusion echo thresholds and localization dominance (via lateralization) for lead-lag click pairs carrying ITD and ILD. Across baseline, buildup, breakdown, buildup retest conditions, lead-lag fusion and localization dominance extended to longer lead-lag delays for stimuli lateralized by ITD than for stimuli lateralized by ILD. Similar observations of different PEs for stimuli lateralized by ITD versus ILD were also made by Saberi and colleagues (see Saberi and Antonio 2003; Saberi et al. 2004) and by Stecker and Brown (2010, 2012) and Brown and Stecker (2010, 2011). Thus, headphone studies which manipulate ITD exclusively (including a majority of the studies discussed hereto) may fail to simulate effects observed in the free field, where ILD apparently makes a significant contribution to the potency of post-onset spatial information (e.g., Stecker and Hafter 2002; Stecker et al. 2013).

Effects of Lead-Lag Spatial Separation. Some studies have demonstrated that the PE is more robust for lead and lag stimuli located in spatial proximity to one another. Litovsky and Shinn-Cunningham (2001) demonstrated that lag discrimination suppression was stronger for lead and lag stimuli carrying similar or identical ITDs than for stimuli carrying different ITDs (cf. Shinn-Cunningham et al. 1993); Brown and Stecker (2013) found that localization dominance and fusion echo thresholds were greater when lead and lag ITD or ILD were restricted to a single hemifield than when the cues were mirror opposite. Interestingly, that effect was greater for ILD than ITD stimuli, with ILD-based fusion echo thresholds increasing by $\sim 3 \mathrm{~ms}$ for "within hemifield" stimuli compared to "opposite hemifield" stimuli. Effects of lead-lag spatial separation have also been investigated considerably in animal models and are discussed further in the "Comparative Studies" section.

Precedence Effects Without Interaural Differences. To investigate whether the PE is exclusive to stimuli that manipulate binaural information, a small number of 
studies have assessed aspects of the PE using "monaural" stimuli-signals presented either to only one ear, or in the midsagittal plane, such that interaural differences were not varied. In general, these studies have suggested that some, but not all, aspects of the PE occur to a similar extent for binaural and monaural stimuli. Rakerd et al. (2000) measured fusion echo thresholds using running speech stimuli presented from two different locations in either the horizontal plane (binaural condition) or midsagittal plane (monaural condition). Though the authors used an unconventional definition of echo threshold-the relative lag intensities that produced just-perceptible second images across a fixed set of lead-lag delays - their data reinforced earlier reports (see Litovsky et al. 1999) that the fusion echo threshold is similar for monaural and binaural stimuli. Agaeva and Al'tman (2008) reported that subjective fusion might even be somewhat greater for longduration (e.g., $100 \mathrm{~ms}$ ) stimuli in the midsagittal plane than in the horizontal plane. In terms of localization dominance, however, the PE appears to be weaker in the midsagittal plane than in azimuth. Dizon and Litovsky (2004) measured localization dominance (values of $c$, Shinn-Cunningham et al. 1993) for leadlag stimuli in the frontal vertical plane and found that, even at 2-ms lead-lag delay, the lag significantly influenced localization judgments. Similarly, Macpherson and Wagner (2008) found in a VAS study that localization of brief pulse trains in the midsagittal plane was strongly biased by post-onset information even at brief interpulse intervals, suggestive of weak localization dominance (cf. Stecker and Hafter 2002). Finally, Bianchi et al. (2013) measured fusion echo thresholds for lead-lag transients presented over earphones either monaurally or binaurally. Echo thresholds were similar ( $~ 5 \mathrm{~ms})$ in the two conditions, with no significant group-level differences across their six subjects. Data thus suggest that some aspects of the PE (e.g., lead-lag fusion) occur similarly for binaural and monaural stimuli, suggestive of monaural or prebinaural processing (e.g., Hafter et al. 1988; Wickesberg and Oertel 1990; Braasch 2013; Bianchi et al. 2013), while others (e.g., localization dominance) do not.

\section{Studies of the Precedence Effect in Special Populations}

Precedence Effects in Children. Studying the developmental time course of the PE offers to provide insight on (1) physiological mechanisms that may underlie the effect and (2) environmental considerations relevant to children (e.g., the design of classrooms). Past studies demonstrated significantly longer echo thresholds for children aged $\$ 5$ years $(25-45 \mathrm{~ms})$ than for older children or adults ( $<15 \mathrm{~ms}$; Clifton et al. 1984) and greater discrimination suppression for children (both 5-year olds and 18-month olds) than for adults (Litovsky 1997), based on azimuthal lag discrimination thresholds. More recently, Litovsky and Godar (2010) examined PEs in 5-year-old children and adults using both fusion and localization tasks with a large range of lead-lag delays (5-100 ms). Consistent with earlier studies, children exhibited significantly longer echo thresholds than adults. In the localization task, children also exhibited poorer localization of the lead and lag signals over an extended range of lead-lag delays. A contributor to this deficit appeared to be increased "temporal order confusion" (localization of the lag when instructed to localize the lead, and viceversa; cf. Stellmack et al. 1999). Thus, although the fusion aspect of the PE would appear to be more robust among children than adults, a lack of strong localization dominance accompanying longer fusion echo thresholds and a greater propensity to confuse lead and lag signals beyond the temporal limit of fusion might instead be taken to suggest generally immature spatial hearing in children (cf. Clifton et al. 1984; Litovsky and Godar 2010).

Precedence Effects in People with Hearing Loss. A number of investigations over the past two decades have assessed the PE in people with sensorineural hearing loss (e.g., Cranford et al. 1993; Goverts et al. 2002; Roberts et al. 2002, 2003; Roberts and Lister 2004; Lister and Roberts 2005; Akeroyd and Guy 2011). While data have been widely variable, hearing-impaired individuals clearly experience both fusion (e.g., Roberts et al. 2002; Roberts and Lister 2004, 2004) and localization dominance (Cranford et al. 1993; Goverts et al. 2002; Akeroyd and Guy 2011). The effect of localization dominance does appear to be weaker in hearing-impaired listeners than in normal-hearing individuals (Cranford et al. 1993; Goverts et al. 2002) and can be predicted to a degree by the severity of hearing loss (Akeroyd and Guy 2011), but it remains unclear whether this effect is attributable to a reduced PE or to reduced localization accuracy in general.

Precedence Effects in Users of Cochlear Implants. Electrical stimulation of auditory nerve fibers with cochlear implants (CIs) offers to restore hearing-including, in the case of bilateral CIs, binaural hearing - in deaf and profoundly hearingimpaired individuals. Unfortunately, performance in "binaural" tasks, including sound localization, remains poor in most bilateral CI users (e.g., Litovsky et al. 2006; Grantham et al. 2008). A certain constraint on performance is that the devices do not preserve temporal information carried by the input signal (e.g., most devices provide no temporal fine structure 
cues and are not synchronized across the ears), which particularly limits sensitivity to ITD. Poorer ITD sensitivity within individual subjects is associated with worse localization in reverberation (Kerber and Seeber 2013), suggesting in turn that the PE, which strongly depends on ITD cues, may be abnormal in users of bilateral CIs. Studies by Seeber and Hafter (2008) and Agrawal (2008) have suggested that precedence is indeed abnormal among bilateral CI users, with widely variable fusion echo thresholds (Seeber and Hafter 2008) and relatively weak localization dominance and lag suppression (Agrawal 2008). In contrast, under direct stimulation, a procedure by which the binaural information provided by bilateral CIs can be precisely controlled via custom research processors, the PE appears to be more intact. Data reported by van Hoesel (2007) and Agrawal (2008) suggested qualitatively similar discrimination suppression in bilateral CI and normal-hearing listeners, at least among bilateral CI users with adultonset deafness (Agrawal 2008; cf. Litovsky et al. 2010). In a more recent study, Brown et al. (2013) found that bilateral CI users experienced both lead-lag fusion and localization dominance, though localization dominance was more variable among CI users than among normal hearing listeners.

Precedence Effects in Individuals with Neurological Impairments. A few studies have evaluated aspects of the PE in individuals with neurological impairments. To the degree that the specificity of pathology is understood, these studies offer to provide insight on brain structures that may be required for normal PEs. Cranford et al. (1990) measured lead localization in a population of individuals with multiple sclerosis and a group of normal controls. Individuals with multiple sclerosis exhibited worse-than-normal performance at lead-lag delays $<1 \mathrm{~ms}$, but not (on average) at longer lead-lag delays, suggestive that pathological demyelination may disrupt precedence at brief lead-lag delays. In a case study, Litovsky et al. (2002) evaluated the PE, in terms of free-field lead-lag fusion and headphone lag ITD discrimination, in an individual with a focal lesion of the right inferior colliculus and lateral lemniscus. In addition to impaired lead-alone localization of stimuli in the left hemifield (i.e., sounds contralateral to the lesion), the subject exhibited reduced lead-lag fusion for left-lead, right-lag stimuli and reduced discrimination suppression (i.e., better discrimination of changes in lag ITD), relative to normal control subjects. These data led the authors to conclude that an intact inferior colliculus is necessary for a normal PE. Finally, Mickey and Dalack (2005) studied the PE in individuals with schizophrenia, using a free-field fusion task. No significant differences were found between schizophrenic and normal control subjects, indicating that schizophrenia, thought to relate (at least in part) to deficits in thalamic function (e.g., Andreasen 1997), does not compromise the fusion aspect of the PE.

\section{Human Physiological Studies}

Human physiological measures offer to provide valuable insight on the biological substrates of PE phenomena observed psychophysically. Recent studies have utilized a variety of electrophysiological measures, including auditory brainstem responses (ABR; Liebenthal and Pratt 1999; Damaschke et al. 2005; Bianchi et al. 2013), middle latency responses (MLR; Liebenthal and Pratt 1999), cortical auditory evoked potentials (CAEP; Dimitrijevic and Stapells 2006; Sanders et al. 2008, 2011; Spierer, et al. 2009; Backer et al. 2010; Bishop et al. 2012), and mismatch negativity (MMN; Damaschke et al. 2005; Dimitrijevic and Stapells 2006). One study additionally compared psychophysical data to ABR measures and clickevoked otoacoustic emissions (CEOAE) originating in the inner ear (Bianchi et al. 2013).

Results of these studies support a monaural peripheral origin of some aspects of the PE occurring at short lead-lag delays (roughly 1-5 ms). Damaschke et al. (2005) and Bianchi et al. (2013) both reported reductions in the amplitude of ABR elicited by the lag click for short delays (maximally 1-2 $\mathrm{ms}$ ) that were not dependent on the binaural configuration of the stimulus. CEOAE amplitudes (Bianchi et al. 2013) demonstrated similar suppression over a somewhat greater range of delays (largest for 1-4 ms). Because the CEOAE is thought to reflect the mechanical response of the basilar membrane rather than the physiological effects of neuronal or hair-cell activity, Bianchi et al. (2013) argued that the peripheral suppression responsible for PE at short delays originates in lead-lag interactions on the basilar membrane itself (cf. Tollin 1998) rather than in adaptation of hair-cell or neural responses (Hartung and Trahiotis 2001) or recovery times of auditory nerve or brainstem responses (see Fitzpatrick et al. 1999).

Liebenthal and Pratt (1999) also measured ABR for lead-lag click pair stimuli. In that study, binaural lag responses were compared to sums of monaural lag responses. Consistent with a monaural origin of ABR correlates of the PE, Liebenthal and Pratt (1999) noted no consistent specifically binaural effects on ABR components. In contrast, binaural echo suppression was reliably observed in the auditory cortical MLR component $\mathrm{Pa}$ ( 30-ms latency). This suppression was observed across lead-lag delays (ranging up to $20 \mathrm{~ms}$ ) and significantly correlated with psychophysical lateralization of the stimuli. Thus, the authors argued in favor of primary cortical involvement in the 
$\mathrm{PE}$, especially in the critical range of 4-12 ms, where peripheral contributions appear negligible.

Other support for a key role of cortical processing in the PE comes from studies of late evoked potentials. Several studies have demonstrated reliable differences in the auditory cortical N1 component (latency around $100 \mathrm{~ms}$ ) depending on whether lead-lag stimuli give rise to fused or nonfused images (Sanders et al. 2008; Spierer et al. 2009; Backer et al. 2010). Sanders et al. (2008) presented click pairs from loudspeakers arranged $55^{\circ}$ left and right of midline, whereas Backer et al. (2010) presented similar stimuli over headphones using VAS techniques. In both studies, a large number of trials were presented at delays close to each listener's echo threshold. Subjects judged the spatial fusion on each trial, and EEG recordings were analyzed in groups of trials on which subjects did or did not report a fused percept. Sanders et al. (2008) reported a significant negative potential (the "object-related negativity" or ORN; Alain et al. 2001) occurring 100-250 ms after onset on trials in which a second (lag) image was perceived. Backer et al. (2010) similarly demonstrated significant differences in CAEP components $\mathrm{N} 1$ and P2 for trials in which the lag image was or was not perceived. Spierer et al. (2009) used a somewhat different approach to demonstrate topographic changes in EEG responses to fused versus segregated click pairs carrying ITD or ILD. Such differences were significant during the 70-117-ms latency range. Thus, all three studies appear consistent with PE-dependent modulations of CAEP in the latency range of component N1 and later.

Damaschke et al. (2005) presented an "oddball" paradigm in which diotic reference click pairs were presented every $500 \mathrm{~ms}$, replaced by "deviant" pairs (which featured a lag ITD) on $12 \%$ of trials. The MMN response, an enhanced negativity that follows deviant stimuli, serves as a marker of change detection. In the case of Damaschke et al. (2005), MMN amplitude indexed the listeners' perception of change in lag location and accurately predicted listeners' psychophysical lag-discrimination thresholds for leadlag delays ranging 1-20 ms. This was not the case for ABR measurements made in the same study. Thus, the authors concluded that the PE is not the result of peripheral distortions but that a physiological correlate of the PE in humans is observed in the (cortical) MMN.

Importantly, many of the studies mentioned above presented stimuli that either intentionally or unintentionally evoked PE buildup effects. Backer et al. (2010) preceded stimuli with a train of 11 conditioning click pairs identical to the final (test) click pair. Dimitrijevic and Stapells (2006) and Spierer et al.
(2009) both compared CAEP for click pairs presented early versus late in a conditioning train to study $\mathrm{PE}$ buildup directly. Both studies demonstrated changes in the N1 latency range: Dimitrijevic and Stapells (2006) reported N1 amplitude reductions following buildup, while Spierer et al. (2009) demonstrated buildup-related shifts in EEG scalp topography consistent with the fused-versus-segregated differences they observed overall. Interestingly, that result occurred only when clicks carried ITD; when ILD was the cue, the patterns did not evolve over time but instead depended mainly on the spatial configuration of the stimulus (see below).

Sanders et al. (2011) compared CAEP measurements for click pairs presented in a buildup paradigm (seven conditioning pairs identical to the test pair) and in a "depressed buildup" condition where the conditioning train was preceded by five clicks from a single loudspeaker. Psychophysically, buildup enhanced the likelihood of fusion relative to the depressed buildup condition, which was similar to the baseline condition (click pairs presented in isolation). Buildup also reduced the magnitude of ORN (Sanders et al. 2008) relative to depressed buildup, consistent with ORN as a marker of segregated perception of the lag-click image.

Finally, two studies reported significant hemispheric asymmetries in the electrophysiological markers of the PE. The results were similar in each case: Dimitrijevic and Stapells (2006) reported greater reductions of lag N1 amplitude for right-lead, left-lag click pairs. Similarly, Spierer et al. (2009) reported EEG topography to more closely follow the "fused" pattern for right-lead, left-lag click pairs, although only when ILD was the manipulated binaural cue. Both results are consistent with psychophysical results demonstrating stronger fusion for right-leading than left-leading click pairs (Grantham 1996; Saberi et al. 2004), particularly under buildup conditions (Grantham 1996). Interestingly, such asymmetry has only been reported for stimuli carrying ILD. Grantham (1996) and Dimitrijevic and Stapells (2006) presented sounds from loudspeakers, whereas Saberi et al. (2004) presented sounds over earphones with ILD as the cue. Spierer et al. (2009) observed the asymmetry for stimuli carrying ILD but not ITD (cf. Saberi et al. 2004).

\section{Summary}

1. The PE consists of several different perceptual phenomena that generally but do not necessarily co-occur. At sufficiently brief lead-lag delays (dependent on a multitude of paradigm, stimulus, and 
subject factors), listeners tend to localize a spatially and temporally dispersed acoustic signal at a single, punctate location that more strongly reflects the spatial cues carried by the first-arriving signal components than the spatial cues carried by laterarriving components.

2. The PE appears to be dominated by low-frequency ITD information and is most robust for signals of extended duration and bandwidth. Narrowband signals, high-frequency signals, or signals otherwise lacking robust ITD information generally produce weaker lead-lag fusion, lead localization dominance, and lag discrimination suppression.

3. Some aspects of the PE, perhaps most notably leadlag fusion, are subject to effects of stimulus history, which can apparently increase or decrease their temporal extent. Effects of stimulus history are stimulus-specific, dependent not only on the spatial and spectral attributes of lead and lag but also on the composition of individual spatial cues.

4. Psychophysical PEs observed in human listeners, from discrimination suppression at a few milliseconds lead-lag delay to "built up" lead-lag fusion for repeated speech syllables, have recently been related to an array of physiological events (and their electrophysiological correlates in humans) along the auditory neuraxis, from entirely peripheral (cochlear mechanical) to cortical (a few hundred milliseconds post-signal onset). Data suggest that spatial aspects of the PE have their earliest measurable correlates in cortical evoked potentials occurring at least tens of milliseconds post-onset, although many such measurements have been taken under conditions likely to induce buildup effects. Physiological questions, which can be addressed with greater resolution in comparative studies using animal models, are considered in detail in the following major section.

\section{COMPARATIVE STUDIES}

Several dozen studies over past the 15 years have worked to elucidate the biological mechanism(s) that give rise to the PE. At least two motivations for undertaking such studies may be identified: (1) to understand the origins of perceptual precedence phenomena described in humans, and (2) for more basic reasons, i.e., to understand spatial hearing neuroethologically. Despite the morphological and functional diversity of hearing mechanisms across species, precedence-like phenomena have been described in a great variety of auditory models, including primates and other mammals, birds, amphibians, and even invertebrates including crickets and flies. In the following sections, we consider a variety of recent psychophysical and physiological studies in animal models.

\section{Psychophysical Studies}

Psychophysical paradigms employed in animals are fundamentally similar to those used in human listeners. Leading and lagging stimuli are typically presented from oppositely positioned loudspeakers or via earphones (see Fig. 5). Discrimination studies assess the ability of animals to detect changes in the source locations of the lead or lag stimuli but do not require the animals to explicitly localize the sounds (see Moore et al. 2008 for discussion of the differences between absolute and relative measures of sound localization abilities). Localization studies, in contrast, require the animals to indicate where in space the sounds were perceived (e.g., via head or eye movements). One difference in animal (versus human) studies is that fusion, a subjective percept, must be inferred from responses in either discrimination or localization tasks.

Discrimination Studies. Many of the classic PE discrimination studies in animals (e.g., Kelly 1974; Cranford 1982; Keller and Takahashi 1996) were summarized by Litovsky et al. (1999). Briefly, like humans, animals can discriminate left from right for single source (lead alone) stimuli with near-perfect accuracy. For lead-lag stimuli, performance changes systematically with lead-lag delay (e.g., Fig. 5b): At lead-lag delays $<1 \mathrm{~ms}$, discrimination performance is near chance, consistent with perception of a phantom source near the midline, i.e., summing localization. At lead-lag delays between approximately 1 and $10 \mathrm{~ms}$, animals again correctly discriminate the side of the leading source with near-perfect accuracy, i.e., with little impact from the lagging source. Finally, for leadlag delays increasing beyond $\geq 10 \mathrm{~ms}$, the animals' performance declines toward chance, suggesting the perception of two sources, one on each side, and thus a bimodal distribution of responses rather than consistent responses toward the lead. The lead-lag delay at which performance begins to decline has often been interpreted as a discrimination-based echo threshold, as described by Litovsky et al. (1999). Several more recent investigations also employed discrimination methods (gerbils, Wolf et al. 2010; budgerigars, Dent and Dooling (2003a, b); owls, Spitzer et al. (2003); Nelson and Takahashi (2008); bats, Schuchmann et al. (2006); ferret, Tolnai et al. (2014)). These studies have reported, consistent with studies in other species including humans, that animal subjects are generally insensitive to lag spatial information at lead-lag delays less than $\sim 10 \mathrm{~ms}$ (e.g., Spitzer et al. 2003), although there appear to be some 
A
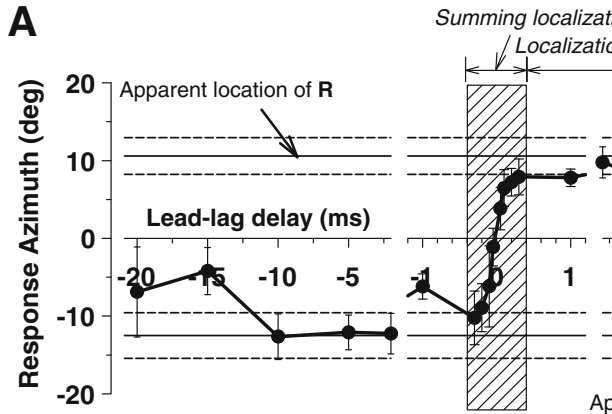

FIG. 5. Cats experience summing localization and localization dominance. A Mean response azimuth $( \pm 1$ SD) for one cat for paired lead-lag stimuli delivered from loudspeakers $L$ and $R$ as a function of the lead-lag delay (filled circles and error bars) and for single stimuli from each location (solid and dashed horizontal lines). Positive delays indicate that source $R$ was leading source $L$, negative delays indicate that $L$ was leading $R$. The hatched region $( \pm 400 \mu \mathrm{s})$ demarcates the temporal extent of summing localization. Localization dominance extends from $400 \mu$ s to $\sim 10$ ms. A localization-based echo threshold of $\sim 10 \mathrm{~ms}$ can be inferred from the responses: At lead-lag delays $>10 \mathrm{~ms}$, the cats

interspecies differences (e.g., bats, Schuchmann et al. 2006; dolphins, Zaslavski 2008).

Localization Dominance. While discrimination paradigms provide some information about spatial perception (e.g., left versus right) and are possible in a wide variety of species, assessment of relative localization ability does not provide information about localization dominance per se. Over the past decade, barn owls, ferrets, and cats-predators that are naturally inclined to orient their gaze toward sources of sound (e.g., Tollin et al. 2009)—have emerged as key models for comparative studies of localization dominance. Figure 5 illustrates an example psychophysical paradigm for assessment of localization dominance in cats; similar paradigms have been used in barn owls (Keller and Takahashi 1996) and nonhuman primates (Populin 2006). Data demonstrate that the animals experience summing localization at lead-lag delays $\leq 1 \mathrm{~ms}$ and localization dominance for lead-lag delays in the range from $\sim 1$ to $10 \mathrm{~ms}$ (Fig. 5), similar to the range over which localization dominance is effective in humans (Fig. 3; cf. Litovsky and Shinn-Cunningham 2001).

Effects of Stimulus Parameters. Several stimulus factors shown to influence the strength and temporal extent of localization dominance in humans also affect localization dominance in animals. For example, in the cat, Dent et al. (2009) found that changes in the spatial separation of lead and lag stimuli systematically changed the lead-lag delays over which localization dominance was experienced. In a free-field task, cats exhibited localization dominance to significantly longer lead-lag delays (up to $\sim 13-26 \mathrm{~ms}$ ) for small azimuthal speaker separations $\left(20-40^{\circ}\right)$ than for larger speaker separations ( 7 ms for speaker separa-

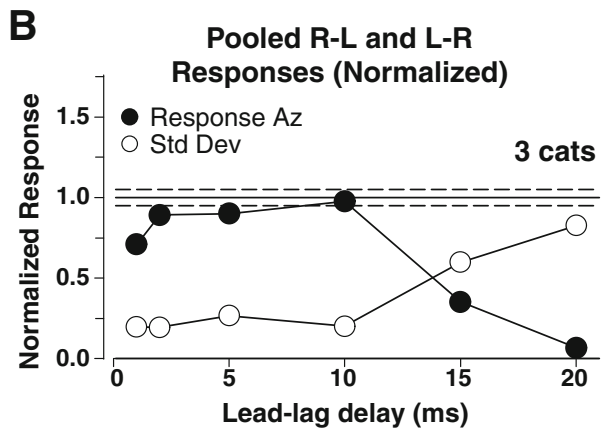

localized the lagging source on $\sim 50 \%$ of trials, which shifted the mean response azimuth toward $0{ }^{\circ}$ and increased its variance. B Mean normalized response azimuth (filled circles) and mean standard deviation of response (open circles) for three cats as a function of lead-lag delay. Solid horizontal and dashed line show mean normalized response \pm 1 SD to a single source at the leading location. Echo threshold was operationally defined as the lead-lag delay at which the behavioral response azimuths trended toward $0^{\circ}(\sim 10 \mathrm{~ms})$. Data replotted from Tollin and Yin (2003).

tions of $\left.120-160^{\circ}\right)$. In a study in gerbil, Wolf et al. (2010) used a two-source discrimination paradigm with narrowband transient stimuli to demonstrate that low-frequency stimuli yielded longer echo thresholds and (inferred) more persistent localization dominance than higher-frequency stimuli, (cf. ShinnCunningham et al. 1995, 1995; Tollin and Henning 1998, 1999; Dizon and Colburn 2006). Finally, Tolnai et al. (2014) recently demonstrated that ferrets experience correlates of the buildup and breakdown effects reported in humans: Like humans, ferrets persist in lateralizing a lead-lag stimulus toward the side cued by the lead over a broader range of lead-lag delays when the test stimulus is preceded by a sequence of identical conditioner stimuli, but not when the conditioner stimuli are spatially reversed (relative to the test).

Lead-Lag Fusion. Fusion is inherently subjective in nature and is typically self-reported by human listeners. Fusion in animal subjects must be inferred on the basis of objective measures. For example, Tollin and Yin (2003) compared the latencies of behavioral responses for the single- and paired-source conditions under the premise that unitary, spatially compact images given by fusion should result in shorter response latencies than when two images were perceived. Specifically, the authors assumed that response latency was correlated with the cats' uncertainty of the source location, with longer latencies expected for those source locations that were more ambiguous (Luce 1986; cf. Gai et al. 2013; Tollin et al. 2013). Consistent with this hypothesis, Tollin and Yin (2003) found that response latencies for paired-source stimuli at brief lead-lag delays were comparable to single-source latencies, whereas latencies were longer 
at lead-lag delays ostensibly beyond the temporal limit of fusion. The data do not prove, however, that cats experienced a fused image like that reported by human listeners in similar tasks.

Somewhat different data were reported by Spitzer and Takahashi (2006) for barn owls: In their study, the latencies of responses to lead-lag stimuli were always $\sim 50 \%$ longer than those to single source stimuli, suggesting that although the owls experienced localization dominance, their certainty about the source location was always reduced by the presence of a lag. Tolnai et al. (2014) reported similar findings in ferrets. Species differences as well as stimulus or methodological differences may have contributed to the difference between these and results obtained in cats. In particular, Tolnai et al. (2014) employed a discrimination paradigm, and Spitzer and Takahashi (2006) used long-duration noise burst stimuli in which lead and lag overlapped in time; the consequences of which have been explored considerably by Nelson and Takahashi (2008, 2010); see below).

Effects of Lead-Lag Temporal Overlap. Nelson and Takahashi (2008) used a novel stimulus paradigm (later employed by Donovan et al. 2012 in humans) to explore which aspects of the lead-lag stimulus control the perceptual salience of the lag. By artificially lengthening the lag-alone segment independent of the lead-lag delay, the authors demonstrated that the proportion of trials on which the owls oriented toward the lag location increased with increasing length of the lag-alone segment. This result was indicative, in their view, that delay-dependent "lag suppression" is a misnomer; the perception, or at least the localizability, of the lag depends on the fidelity of its internal representation, which improves as its duration is increased.

\section{Physiological Studies}

Psychophysical PE studies conducted in animal models have systematically mapped out the ranges of lead-lag delays over which various PE phenomena are experienced by a variety of species (most especially the cat and barn owl). These data are essential toward the interpretation of physiological studies of similar phenomena, for which the goals are to elucidate (1) where along the auditory neuraxis neural correlates of the various PE phenomena first emerge and (2) what mechanisms (e.g., neural adaptation or synaptic inhibition) produce these effects.

A Cautionary Note-Effects of Anesthesia. Many early physiological studies of PE were conducted using animals under general anesthesia (see Litovsky et al. 1999 for review). It has since been established that anesthetics (particularly sodium pentobarbital) can significantly increase neural "lag response" recovery times. For example, while lead-lag delays that produce "half-recovered" responses to the lag in neurons of the inferior colliculus (IC, Tollin et al. 2004; Fitzpatrick et al. 1995) and auditory cortex (Fitzpatrick et al. 1999) of awake animals are $~ 10$ and $\sim 20 \mathrm{~ms}$, respectively, these values increase to $\sim 35$ $\mathrm{ms}$ in the IC (Yin 1994; Litovsky and Yin 1998a, b) and $\sim 100 \mathrm{~ms}$ in the cortex (Reale and Brugge 2000; Mickey and Middlebrooks 2005) of anesthetized animals. Song et al. (2011) studied the effects of sodium pentobarbital anesthesia on neural PE responses directly by measuring the responses of IC neurons in rats before and after drug administration. As the dose of anesthetic increased, the lead-lag delay at which the responses recovered systematically increased. In sum, the effects of anesthesia in physiological studies of the PE can be profound. The majority of review to follow will thus focus on studies that have used unanesthetized animals, though some data from anesthetized animals will be highlighted where appropriate.

Physiological Study of the Precedence Effect-an Example. Figure 6 illustrates an example free-field physiological paradigm for study of the $\mathrm{PE}$, based on data collected by Tollin et al. (2004) in the IC of the unanesthetized, behaving cat. In this example, neural responses to transient signals presented from single source locations are first measured (Fig. 6A) in order to determine the neurons' "spatial tuning," which can be summarized by plotting the response, in numbers of action potentials (or "spikes") per stimulus (or per second), as a function of the spatial location (azimuth/elevation) of the sound source. In most studies, neural responses to presented stimuli are computed within a short window of time shortly after stimulus onset (see "Analysis window" in Fig. 6A). As is the case for most spatially tuned neurons in the IC and auditory cortex (not shown, see Mickey and Middlebrooks 2001), neural responses are greater for contralateral stimuli (speaker $R$ in Fig. 6A) than for ipsilateral stimuli (speaker $L$ in Fig. 6A). After responses are established for single stimuli at these two locations, a lead-lag ( $\mathrm{L}-\mathrm{R}$ or $\mathrm{R}-\mathrm{L}$ ) stimulus is presented to elicit the PE. The order of stimulus presentation is typically varied across trials.

At the most basic level of analysis, neural responses to the leading and lagging sources are quantified using the analysis windows determined from their corresponding single-source responses but adjusted in time to account for the lead-lag delay (Fig. 6B). Lag source response recovery functions are often plotted as a ratio of lag response to lag-alone response at a given location, measured across a range of lead-lag delays to determine the range of lead-lag delays yielding reduced lag responses (e.g., Fig. 6B, Inset). 

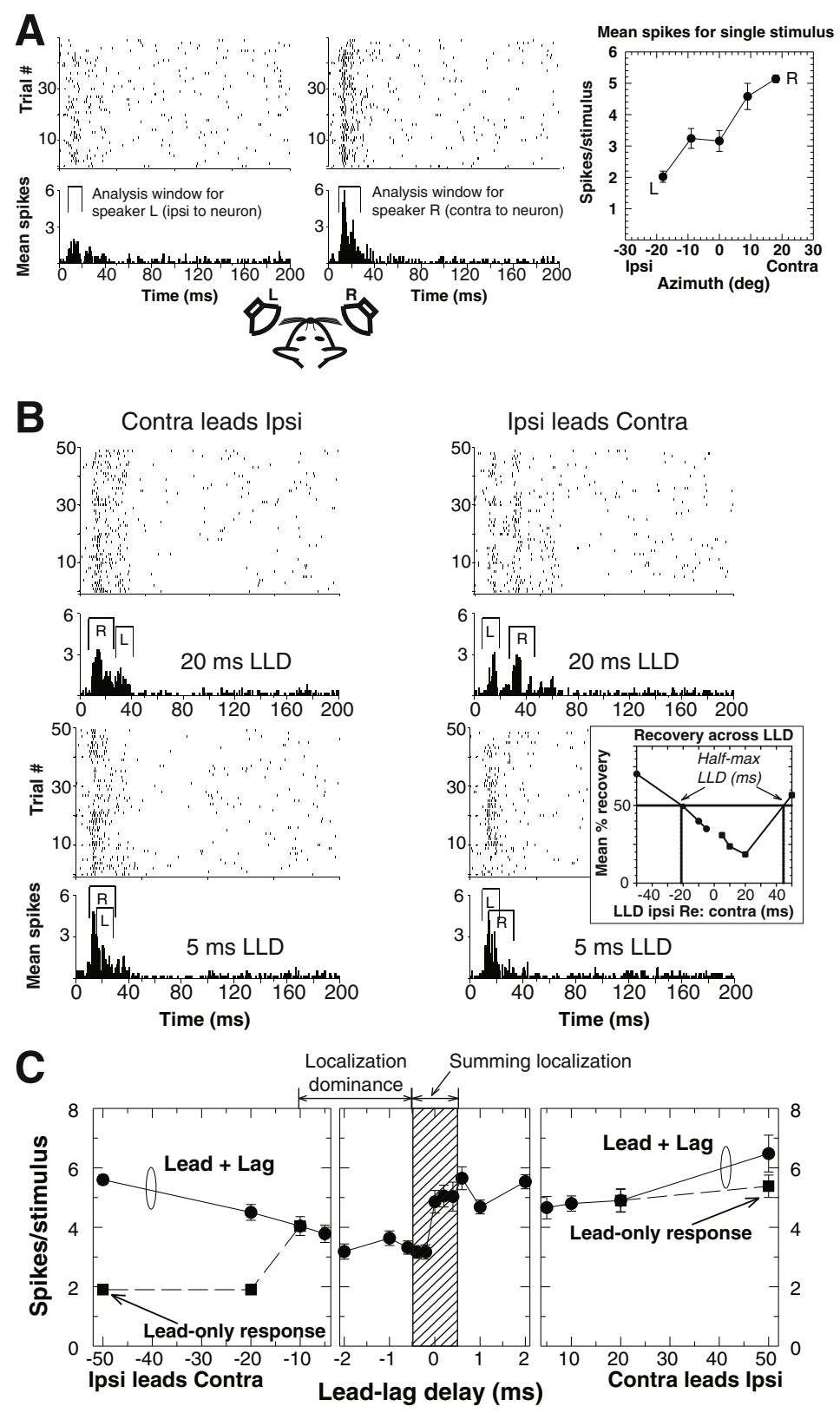

FIG. 6. Example of physiological responses to precedence effect stimuli. A Dot rasters (top panels) and histograms (bottom) of the responses of one IC unit to single sources at two different azimuths $\left(+18^{\circ}[\mathrm{R}]\right.$ or $\left.-18^{\circ}[\mathrm{L}]\right)$, recorded over many trials. For each source azimuth, the response (mean spikes/stimulus) was computed using an analysis window, designed to capture only stimulus-elicited (i.e., acoustically driven) spikes; the analysis window is computed separately at each azimuth as the time window containing mean spikes greater than 2 standard deviations above the spontaneous rate. Mean number of spikes/stimulus ( \pm 1 SEM) is given as a function of sound source azimuth in the right panel. Responses are clearly modulated by variations in source azimuth, with a strong contralateral bias. B Responses of the neuron depicted in $\mathbf{A}$ to paired sources at two lead-lag delays (LLDs) when either the contralateral source (speaker $R$ ) was leading the ipsilateral source (speaker $L$, left column) or vice versa (right column). The total response of the unit was computed either by summing nonoverlapping responses (at lead-lag delays $\geq 20 \mathrm{~ms}$ ), or using a composite analysis window created by merging the analysis windows for the leading and lagging source (e.g., 5-ms delay; see Parham et al. 1996). (Inset) Normalized response to the lag as a function of lead-lag delay for the two pairedsource conditions. "Half-maximal" lead-lag delay indicates the delay at which the neuron's response to the lag recovers to $50 \%$ of its response to a single stimulus ("lead alone") from the same location. The half-maximal delay is measured separately for contraipsi and ipsi-contra lead-lag stimuli (and occurs at substantially different absolute values of lead-lag delay in this neuron). $\mathbf{C}$ Mean number of total spikes per stimulus as a function of lead-lag delay. IC neuronal responses were systematically modulated by lead-lag delay, particularly for lead-lag delays between -10 and $+10 \mathrm{~ms}$. Consistent with behavioral modulation of responses (e.g., Fig. 5A), over this range of lead-lag delays, the neural response was greater when the delays favored the contralateral source (speaker $R$ ) than when they favored the ipsilateral source (speaker $L$ ), with a gradual transition near $0 \mathrm{~ms}$ (i.e., in the region of summing localization). For both ipsi-contra and contra-ipsi stimuli, discrete responses to the lead are evident at sufficiently long lead-lag delays (squares) and comparable in number of spikes to the single stimulus values plotted in A. Data replotted from Tollin et al. (2004). 
Neural Responses to Lagging Sounds Are Weakly Suppressed in Lower Auditory Centers. Variants of the lead-lag paradigm depicted in Figure 6 have been used to study virtually all stations in the ascending auditory pathway, including the auditory nerve (AN; Parham et al. 1996), anteroventral cochlear nucleus (AVCN; Wickesberg 1996; Fitzpatrick et al. 1995; Parham et al. 1998), superior olivary complex (SOC; Fitzpatrick et al. 1995), dorsal nucleus of the lateral lemniscus (DNLL; Pecka et al. 2007), IC (Carney and Yin 1989; Fitzpatrick et al. 1995; Yin 1994; Litovsky and Yin 1998a, b; Burger and Pollak 2001; Tollin et al. 2004; Spitzer et al. 2004; Nelson and Takahashi 2008; Dent et al. 2009; Song et al. 2011), and auditory cortex (AC; Fitzpatrick et al. 1999; Reale and Brugge 2000; Mickey and Middlebrooks 2001, 2005) (see schematic diagram in Fig. 7A). The results of these studies are summarized in Figure $7 \mathrm{~B}$, which plots the mean recovery of the lag response (normalized to the lag alone response) in populations of neurons from each stage as a function of the lead-lag delay. For very brief leadlag delays (e.g., $2 \mathrm{~ms}$ ), neuronal responses to the lag are significantly reduced at all sites. As the leadlag delay is increased, each lag recovery function eventually reaches a level comparable to the response elicited when the lagging source is presented in isolation. The lead-lag delay producing $50 \%$ recovery is often used as a measure of the "neural echo threshold" to provide for comparison against behavioral echo thresholds.

While a general trend of lag increasing response recovery with increasing lead-lag delay can be observed for all areas of the auditory system studied thus far, the rate at which neural responses to the lagging sound recovers and, by extension, the neural echo threshold, appears to strongly depend on the level in the ascending auditory system at which the neurons are located. At more peripheral sites on the neuraxis, including the auditory nerve, cochlear nucleus, and superior olivary complex, neurons begin to respond to both the lead and to the lag for lead-lag delays as short as 1-2 ms. By 5-6 ms, responses to the lag are nearly fully recovered (75\% or greater) in each of these areas. Conceivably, the emergence of reliable lag responses at these peripheral levels could provide a basis for psychophysical echo thresholds on the order of 5-6 ms measured with pairs of lead-lag transients in some experiments (see Table 1). However, in many human and animal psychophysical studies, lag discrimination and especially localization responses suggest very little influence of the lag until delays of $8-10 \mathrm{~ms}$ or more (Figs. 3 and 5). These data are difficult to account for on the basis of limited reductions of neural responses observed at those delays in peripheral (or "prebinaural") auditory neurons.

Evidence That the IC Is a Critical Processing Center for the Precedence Effect. The IC is an excellent candidate site for the generation of $\mathrm{PE}$ and specifically spatial $\mathrm{PE}$ phenomena such as spatially dependent discrimination suppression: Most of the neurons comprising the IC are sensitive to spatial location. Figure 6C plots data obtained in a free-field $\mathrm{PE}$ paradigm like that pioneered by Yin (1994), but using unanesthetized cats (Tollin et al. 2004): At small leadlag delays $(< \pm 1 \mathrm{~ms})$, the responses of IC neurons were modulated in a manner consistent with summing localization (cf. Warncke 1941). For lead-lag delays ranging from \pm 1 to $\sim \pm 10 \mathrm{~ms}$, the total response of the IC neurons to the lead-lag stimulus approximated the response to a single source at the location of the lead, with little effect of the lagging source (cf. Spitzer et al. 2004). For lead-lag delays beyond $\sim \pm 10 \mathrm{~ms}$, a separable response to the lag began to emerge (see also Fig. $6 \mathrm{~B})$. The emergence of a separable lead and lag responses was consistent with increasingly robust perception of the lag (a failure of both lead-lag fusion and localization dominance) at delays beyond $\sim 10 \mathrm{~ms}$, measured in the same animals (Tollin et al. 2004).

Several physiological studies have specifically examined the effects of either directly changing the lead/lag source locations (Litovsky and Yin 1998a, b; Litovsky and Delgutte 2002; Tollin et al. 2004; Dent et al. 2009; Spitzer et al. 2004) or manipulating the lead/lag ITD (Yin 1994; Fitzpatrick et al. 1995) on lead and lag responses in IC neurons. The degree of lag suppression has been shown to depend upon the relative location of the leading source, with maximal suppression generally occurring when the lead and lag are colocated or proximate and progressively less suppression occurring for more disparate locations (Litovsky and Yin 1998a, b; Litovsky and Delgutte 2002; Tollin et al. 2004; Dent et al. 2009; Spitzer et al. 2004). Neurons exhibiting this characteristic have been referred to in the literature as "SMAX" neurons (see Litovsky et al. 1999). A smaller proportion of neurons, known as "SMIN" neurons, exhibit stronger suppression when the lead and lag stimuli are spatially disparate (i.e., when the lead stimulus carries cues different from the lag stimulus; e.g., Litovsky and Delgutte 2002). Thus, while proximate lead and lag sources generally lead to stronger inhibition than disparate lead and lag sources, the existence of SMIN neurons may offer a physiological basis for the occurrence of psychophysical precedence across spatial hemifields.

Mechanisms That May Give Rise to Precedence Effect-Like Responses Within the IC. Yin (1994) suggested that a candidate structure to provide delayed spatially dependent inhibition to the IC would be the DNLL 


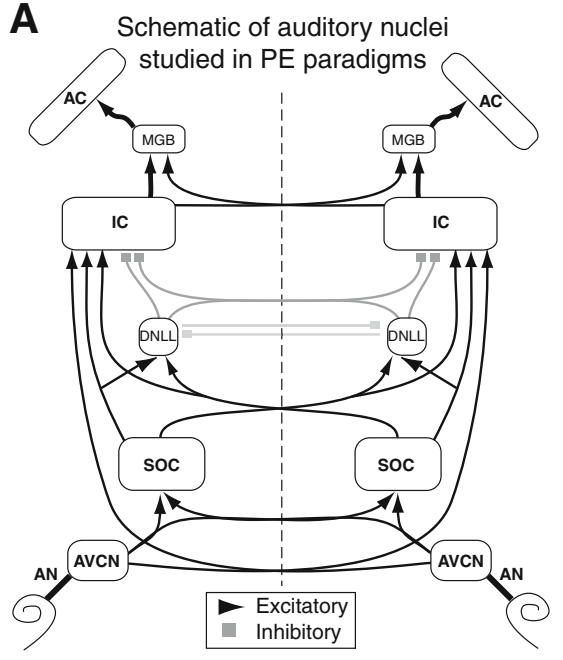

FIG. 7. Neural responses to PE stimuli at sites along the auditory neuraxis. A Schematic diagram of the ascending auditory pathway with an emphasis on groups of neurons (nuclei) studied in physiological PE paradigms. Data for bolded nuclei (AN, AVCN, SOC, IC, AC) are given in B. Detailed anatomy (e.g., multiple nuclei and inhibitory projections within SOC) is not illustrated. B Singleneuron responses to a lagging stimulus are suppressed over a broader range of lead-lag delays at higher levels of the central auditory system. Across-neuron population lag-response recovery functions are given for structures from the auditory nerve $(A N)$ to the auditory cortex $(A C)$. Each data point shows the across-neuron mean proportion recovery $( \pm 1$ SEM, number of neurons is indicated in

(see Figs. 7A and 8). Anatomically, the DNLL projects to both ICs (Hutson et al. 1991; Shneiderman et al. 1988; Shneiderman et al. 1999). Physiologically, DNLL neurons are sensitive to the binaural cues to sound location (Brugge et al. 1970; Kelly et al. 1998; Markovitz and Pollak 1994; Yang and Pollak 1994). Finally, the principal cells of DNLL are GABAergic (Adams and Mugnaini 1984; Gonzalez-Hernandez et al. 1996) and have been shown to provide long-lasting inhibition to IC (e.g., Burger and Pollak 2001; Pecka et al. 2007). DNLL is thus optimally positioned to provide delayed, spatially dependent inhibition to IC neurons over a time course consistent with IC neural lag suppression. Pecka et al. (2007) argued further that DNLL-based inhibition of the IC and of the opposite DNLL (via the commissure of Probst) should lead to impaired lag spatial sensitivity at delays for which lag detection is still possible (consistent with psychophysical disparities between fusion and explicitly spatial measures of the PE; see Pecka et al. 2007). Few other PE studies have empirically examined the role of the DNLL, but it seems that a complete systems-level understanding of the physiological PE will require an understanding of how ipsilateral and contralateral DNLL projections affect empirically measured IC responses. One DNLL-based model
B Recovery of single neuron responses to the lag as a function of lead-lag delay across nuclei

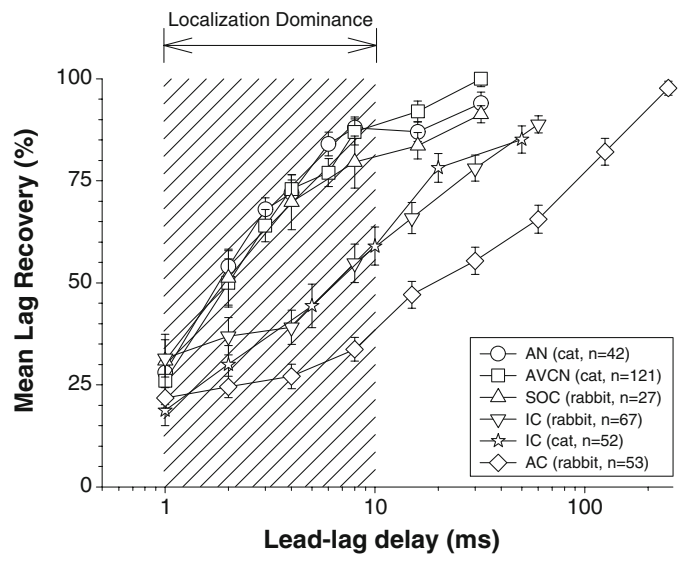

legend) as a function of the lead-lag delay. A value of $100 \%$ indicates that the response to the lag had recovered to the same level as the response to single sound presented from the lagging location. All animals were unanesthetized. There is a significant decrement in lag responses between the SOC and IC, suggestive of a primary role for ICassociated structures in some aspects of the PE (e.g., localization dominance, shaded region; see text). Data were replotted from auditory nerve (AN; Parham et al. 1996), anteroventral cochlear nucleus (AVCN; Parham et al. 1998), superior olivary complex (SOC; Fitzpatrick et al. 1995), inferior colliculus (IC; Fitzpatrick et al. 1995, rabbit; Tollin et al. 2004, cat), auditory cortex (AC; Fitzpatrick et al. 1999).

(Xia et al. 2010) that proposes a biologically plausible explanation for the existence of SMAX and SMIN neurons is discussed in the "Modeling" section (see Fig. 8).

Cortical Involvement in the Precedence Effect. Responses to lagging sources in auditory cortex neurons continue to be suppressed for lead-lag delays well beyond $\sim 10 \mathrm{~ms}$, even in unanesthetized animals (Fig. 7; Fitzpatrick et al. 1999). Using information theoretic techniques, Mickey and Middlebrooks (2005) reported that the vast majority of cortical neurons showed no significant transmission of information about the location of the lagging source for lead-lag delays up to $16 \mathrm{~ms}$, well beyond the $\sim 10$-ms lead-lag delays at which humans, cats, and barn owls first begin to localize the lagging source psychophysically. Thus, on the basis of single unit data, the role of the auditory cortex in the $\mathrm{PE}$ remains uncertain. One piece of evidence against cortical involvement in localization dominance was reported by Tollin et al. (2010): In addition to gaze shifts toward the lead at delays that produce localization dominance, cat's pinnae shift toward the lead and do so with latencies too short to be attributed to cortical processing (see Tollin et al. 2010). It is at present unclear how to reconcile such data with recent human evoked potential studies that argue for an 
A
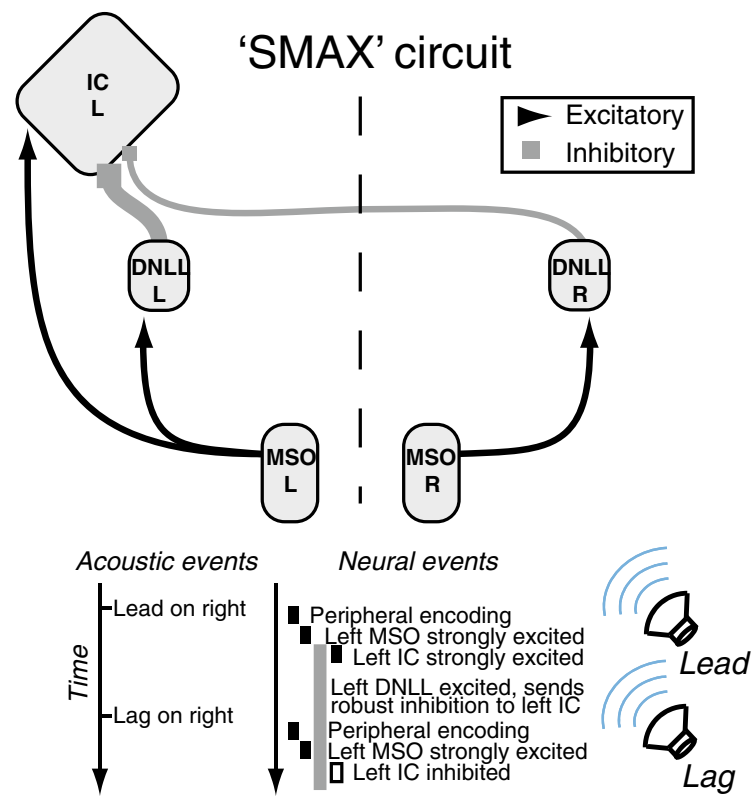

B
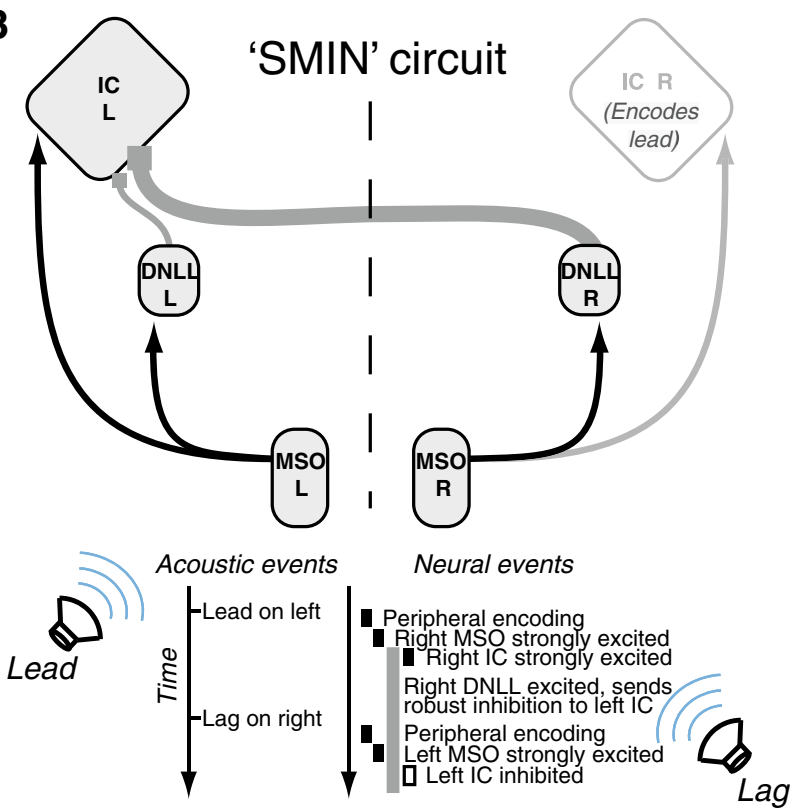

FIG. 8. A biologically plausible role for the DNLL in the PE under different stimulus configurations. A IC neurons receiving robust ipsilateral inhibition from the DNLL should be robustly inhibited for "lag" stimuli when the "lead" is colocated or in the same hemifield. Such neurons, described in the physiological literature as "SMAX" neurons, appear to be ubiquitous in the IC, providing a candidate neural substrate for the inverse relationship between lead-lag spatial separation and the strength of localization dominance. B IC neurons receiving robust contralateral inhibition from the DNLL should be robustly inhibited for "lag" stimuli in the contralateral hemifield when the "lead" is in the ipsilateral hemifield. Such neurons, described in the physiological literature as "SMIN" neurons, appear to be somewhat less common than SMAX neurons but provide a candidate substrate for the persistence of the PE when the lead and lag are spatially disparate. Diagrams adopted from Xia et al. 2010, Figure 1.

important role of long-latency cortical processing in some aspects of the PE, though the presence or absence of conditioner (buildup) stimuli may be an important difference.

\section{Summary.}

1. A variety of experimental animals perform similarly to human listeners when tested in similar or analogous psychophysical PE paradigms. Recent data from predatory animals such as cats, owls, and ferrets that are naturally predisposed to orient toward sound sources suggest that these animals experience true localization dominance, similar to human listeners. Data suggest some interspecies differences (e.g., between cats, owls, and bats). Additional data is needed from other vertebrates and invertebrates to more formally understand the conservation of PE phenomena across species and taxa.

2. Physiological data obtained from experimental animals suggest that some aspects of the PE may emerge at relatively peripheral sites in the auditory neuraxis, while others (e.g., localization dominance) almost certainly require more central processing, such as synaptic inhibition, perhaps in the inferior colliculus via the dorsal nucleus of the lateral lemniscus. It is essential to understand and avoid confounding effects of anesthesia in physiological experiments of the PE, which in earlier studies led to overestimates of its neural time course.

3. While neural mechanisms have been identified in experimental animals that are concordant with psychophysical responses observed in the same animals, the level at which the perception of a single or multiple auditory objects arises, and the manner by which the locations of those objects are ascribed (a very fundamental question), remains unknown. Some data from cats and rabbits suggest PE-like processing in the auditory cortex, but much more data is needed before the role of the cortex in precedence phenomena can be extrapolated.

\section{MODELS OF THE PRECEDENGE EFFECT}

Finally, we consider a number of recent models that have been proposed to account for aspects of the PE. Some of the models to be discussed were developed to account for PE data specifically, while others are more general models that have been tested for their ability to account for the PE. These models vary in their degree of computational detail and biological specificity but can in general be attributed to more "peripheral" or "central" domains. In many models, outputs consist of "internal ITDs" for the lead and/or lag estimated from the cross-correlation of transformed left- and right-ear signals (e.g., hair cell synaptic outputs, or neural spike trains; see Colburn 1973; 
Blauert and Cobben 1978; Stern et al. 1988). In some cases, interaural cross-correlation is assessed in a timevarying manner, which allows for analysis of leading and lagging information available at discrete points in time (e.g., Tollin 1998; Xia and Shinn-Cunningham 2011).

\section{Peripheral Models}

Impinging acoustic signals cause motion of the basilar membrane that can persist for milliseconds after the signal offset, most especially in apical (low-frequency) regions of the cochlea with narrow mechanical tuning. Such "ringing" can lead to a few milliseconds of cyclic auditory nerve fiber output even for impulsive/transient input stimuli (e.g., Kiang et al. 1965). Tollin (1998) proposed that such ringing of the basilar membrane might precipitate precedence-like effects, without invoking central inhibitory mechanisms. For pairs of lead-lag transients, Tollin (1998) modeled the excitation of the basilar membrane and corresponding auditory nerve fibers as the output of narrowband gammatone filters centered near $750 \mathrm{~Hz}$, in the so-called binaural dominance region (e.g., Bilsen and Raatgever 1973; Gaskell 1983; Tollin and Henning 1998). By quantifying the interaural differences in the left- and right-ear filter outputs, Tollin (1998) was able to predict essential features of precedence effects at brief lead-lag delays, including localization dominance, lead-lag ITD "trading" (Wallach et al. 1949), and "anomalous lateralization" in which lateral position judgments for lead-lag stimuli appear opposite those expected based on the lead cues (Tollin and Henning 1998, 1999; Braasch and Blauert 2003; Dizon and Colburn 2006). Thus, Tollin (1998) demonstrated that some aspects of the PE could be accounted for at the most peripheral level of the auditory system, in the ear itself, as a consequence of binaural cue distortion (mainly ITD, but also ILD—see Tollin 1998; Hartung and Trahiotis 2001; Braasch and Blauert 2003).

Hartung and Trahiotis (2001) further explored the notion of peripheral PEs. Using the Meddis (1986) hair cell model, which imposes nonlinear compression and adaptation effects on gammatone filter outputs, the authors computed the outputs of left and right auditory filter banks for lead-lag stimuli and computed their resultant cross-frequency interaural cross-correlation. Taking the effective lateral position as the peak of the average cross-correlation function (after cross-frequency weighting that emphasized lowfrequency information, cf. Tollin 1998), Hartung and Trahiotis (2001) were able to account for PE data obtained at brief lead-lag delays up to $\sim 2-3 \mathrm{~ms}$ (e.g., lead-lag trading data of Wallach et al. 1949; Yost and Soderquist 1984). Xia and Shinn-Cunningham (2011) took a similar approach but extracted peripheral responses as the outputs of an auditory nerve model (Carney 1993) and considered the resultant interaural cross-correlation data over time, with respect to known physiological distributions of neuronal "best ITDs." The authors were able to predict lead-lag ITD lateralization results obtained at 1- and 2-ms lead-lag delay with a variety of stimuli. Brown and Stecker (2012) took a similar approach, applying the auditory nerve model of Zilany et al. (2009), which features "power-law" adaptation, to evaluate peripheral processing effects at longer lead-lag delays. Auditory nerve model outputs replicated auditory nerve data reported by Parham et al. (1996), which demonstrated persistent (but systematically declining) attenuation of lag responses (relative to lead responses) at lead-lag delays of 4, 8, and even $16 \mathrm{~ms}$ (see Fig. 7). In sum, a variety of studies have suggested that some aspects of the PE evident at brief lead-lag delays might be accounted for without resorting to central mechanisms.

\section{Central Models}

Toward an understanding of aspects of the PE that persist to longer lead-lag delays or strongly depend on spatial attributes of the lead and lag, a number of models have been developed that implement mechanisms to explicitly suppress the spatial information carried by the lagging sound, typically within the context of a larger-scale binaural lateralization model. One approach has been to temporarily inhibit binaural information following sound onsets (Lindemann 1986a, b; Zurek 1987). A germinal example is the model of Lindemann (1986a, b), who extended the binaural cross-correlation model to include contralateral inhibitory mechanisms as well as monaural detectors. These modifications allowed Lindemann (1986a) to account for a variety of phenomena including lateralization by interaural level differences, ITD/ILD trading, multiple images resulting from conflicting cues, and effects of varying interaural correlation. In addition, Lindemann (1986b) invoked the contralateral inhibition mechanism to account for localization dominance in bandpass impulse pairs in which the lag carried an ITD, as a function of both lead-lag delay and impulse frequency. In this model, temporary inhibition triggered by the primary crosscorrelation peak suppresses secondary peaks resulting from the lag.

Braasch and Blauert (2003) compared various binaural models' predictions of PEs for lead-lag pairs of temporally overlapping noise bursts. Binaural crosscorrelation and excitatory-inhibitory ("EI") algorithms (cf. Breebaart et al. 2001a, b) were tested with and without inhibition (as in Lindemann (1986a, b) 
model), with an integrated hair cell model (Meddis 1986) as proposed by Hartung and Trahiotis (2001), and with spectral-dominance weighting as proposed by Tollin (1998). While the hair-cell and spectraldominance models were able to account for many aspects of the PE for click pairs presented at short delays, Braasch and Blauert (2003) reported that these models failed to account for PEs with longerduration stimuli, in which lead and lag temporally overlap. The modified Lindemann model was more successful, highlighting the likely role of postperipheral mechanisms (e.g., inhibition) in shaping the PE for real-world stimuli such as speech.

Braasch (2013) implemented a PE model that aimed to reduce the lag representation in each ear's signal prior to binaural comparison. Rather than triggering inhibition at the overall sound onset, the Braasch (2013) model uses autocorrelation to determine the relative amplitude and timing of the lead and lag signals, separately in each ear. This information is then used to derive a finite-impulse-response inverse filter that attenuates the lag in the monaural signal at each ear. Importantly, the model is able to account for PEs in sounds that lack overall onset cues (e.g., Dizon and Colburn 2006; Nelson and Takahashi 2010; Donovan et al. 2012)-instances for which models using onset-triggered inhibition fail. While it is not clear to what extent this model mimics physiological processes, the monaural inverse filtering is in some ways similar to delayed inhibitory circuits described in the cochlear nucleus (e.g., Wickesberg and Oertel 1990).

An alternative to post-onset suppression of binaural information has been to weight or extract the cues occurring only in the periods of the stimulus likely to be dominated by the lead sound. Wolf (1991) extracted ITD from the onset slopes (i.e., regions where the temporal envelope slope is significantly positive), an approach supported by recent psychophysical and physiological evidence for the dominance of binaural cues that occur during periods of increasing envelope slope (Nelson and Takahashi 2010; Dietz et al. 2013; Baxter et al. 2013; Stecker and Bibee 2014). Faller and Merimaa (2004) measured the time-varying interaural coherence of leadlag click trains, noises, and speech sounds in order to detect instants in which the direct sound carries significant energy in a given critical band. Extracting ITD and ILD cues from only those instants enables the model to accurately track lead-sound direction in the presence of delayed copies, reverberation, and spatially competing distracters. For lead-lag click pairs, the cue-selection model of Faller and Merimaa (2004) exhibits all three phases of the PE (summing localization, localization suppression, and independent localization of the lead and lag) as ICI is extended from 0 to $20 \mathrm{~ms}$. A similar approach employed by Dietz et al. (2011) applies cue selection by interaural vector strength within a comprehensive binaural processing model that incorporates envelope and fine-structure processing of ITD and ILD information. That model successfully captures sound-source directions in the presence of noise and reverberation as introduced by binaural room impulse responses.

A combined approach (featuring both onset emphasis and spatial inhibition) was recently described by Takanen et al. (2013), in the form of a comprehensive binaural model based on count-comparison coding. The model is capable of predicting lateralization on the basis of ITD and ILD cues in a variety of stimulus contexts, including simulations of the PE. The model incorporates biologically inspired models of the medial superior olive (MSO) and LSO for ITD and ILD extraction, along with higher-order processes that combine cues across outputs of those pathways, enhance onsets, and provide spatial contrast via contralateral inhibition. Onset enhancement and spatially dependent inhibition in particular provide the model with appropriate predictions of localization dominance at short delays for lead-lag pairs and for the case of multiple reflections embodied in recorded binaural room impulse responses.

A biologically explicit model that features spatially dependent inhibition (based on low-frequency ITD processing) was proposed by Xia et al. (2010). The model features biophysical models of auditory nerve fibers, cochlear nucleus spherical bushy cells and MSO principal cells in series, and inhibitory (GABAergic) DNLL neurons (inverting inputs from MSO) as inputs to each IC (see Fig. 8). For lead-lag input stimuli, effects of peripheral lead-lag interactions occur at the level of MSO (prior to DNLL inhibition) for lead-lag delays to a maximum of $\sim 5 \mathrm{~ms}$. At the level of IC, the model captures the graded recovery of ITD-sensitive neuronal responses to the lag beginning at approximately 10-ms lead-lag delay (cf. Tollin et al. 2004) in a manner that depends, for each neuron, on the spatial locations (ITD) of the lead and lag stimuli and on the relative strength of inputs from contralateral versus ipsilateral DNLL. Specifically, the lag responses of neurons that receive stronger ipsilateral than contralateral inhibition from DNLL (SMAX neurons; e.g., Litovsky et al. 2002) are most suppressed when the lead and lag are colocated (Fig. 8A). Responses in neurons that receive stronger contralateral than ipsilateral DNLL inhibition, conversely, are most suppressed when the lead is located in the opposite hemifield (Fig. 8B). The authors used the model to successfully predict a variety of psychophysical localization dominance data, including estimates of the neural "precedence ratio" ( $c$, ShinnCunningham et al. 1993). The model of Xia et al. 
(2010) is thus broadly consistent with a variety of psychophysical and physiological data discussed hereto, including possible monaural effects at lead-lag delays of approximately $\leq 5 \mathrm{~ms}$, and binaural effects requiring synaptic inhibition at lead-lag delays of $\sim 10$ ms and beyond.

\section{Summary}

1. Aspects of the PE measured at very brief lead-lag delays can be accounted for by models of peripheral auditory processing that do not include explicit mechanisms for "echo suppression." These include fusion, discrimination suppression, and localization dominance at delays shorter than $\sim 3-5 \mathrm{~ms}$, as well as the pattern of delay-dependent anomalous lateralizations that occur for transient and narrowband stimuli in this range. However, aspects of the PE measured at lead-lag delays of 5-10 ms and beyond are not well-accounted for by peripheral models.

2. Models that include explicit mechanisms for lag inhibition have been successful at predicting aspects of the PE that persist to 10 -ms lead-lag delay and beyond. However, some of these models implement onset-triggered inhibition that cannot account for the "ongoing" PEs (e.g., localization dominance in the absence of strong onset cues).

3. Models that extract spatial cues from particular instants of an ongoing stimulus, for example by increased weight on cues occurring during increases in the temporal envelope, may perform similarly to inhibition-based models for stimuli with strong onsets, while better accounting for ongoing precedence effects in longer-duration stimuli. In some cases, such models are well supported by the characteristics of neural behavior.

4. Models that combine peripheral and central processing stages will likely be necessary to account for all aspects of the PE. Similarly, models that integrate ITD, ILD, and spectral shape processing will likely be necessary to capture the full range of behavioral responses to naturalistic $\mathrm{PE}$ stimuli.

\section{GENERAL DISCUSSION}

The PE, first understood as a perceptual phenomenon, has inspired decades of research using diverse psychophysical, physiological, and computational methods. We have attempted to be comprehensive in our coverage of important modern PE findings. In this final section, we briefly consider a few aspects we do not yet understand or clearly understand about the $\mathrm{PE}$, experiments that might fill these gaps in under- standing, and finally, somewhat more philosophically, why the PE and like phenomena may be so ubiquitous.

\section{Extant Gaps in Understanding and Some} Experiments That May Fill These Gaps

Effects of Stimulus Design. Equal-amplitude lead and lag transient stimuli have been used to study the PE literally hundreds of times. These stimuli, though tractable and certainly relatable to a source and single reflection, are highly synthetic. Lag attenuation (e.g., Rakerd et al. 2000; Hafter et al. 2001; Yost 2007) offers a more realistic approximation of natural reflections but does not account for spectral distortions, reverberation, and other effects likely to occur in real environments. Thus, particularly with the widespread availability and computational feasibility of modern virtual space techniques, a productive avenue for future inquiry will be to relate aspects of the classical paired-source PE to PEs measured using ecologically inspired stimuli. Several recent studies (e.g., Devore et al. 2009; Devore and Delgutte 2010; Brandewie and Zahorik 2010; Ihlefeld and Shinn-Cunningham 2011) have used simulated reverberant environments to construct more natural stimuli for psychophysical (e.g., Brandewie and Zahorik 2010; Ihlefeld and Shinn-Cunningham 2011) and physiological (e.g., Devore et al. 2009; Devore and Delgutte 2010) studies. Additional experiments using more naturalistic paradigms will allow specification of stimulus features that do or do not give rise to various aspects of the laboratory lead-lag PE. Studies that use naturalistic stimuli presented over an extended period of time will also work to further elucidate effects of repeated stimulation (buildup and breakdown), their correspondence to paradigms that employ identical onset and post-onset signals, and their relevance to real-world listening. It would be particularly interesting to measure "natural" echo thresholds with respect to the known geometry of various reverberant environments. Everyday experience would suggest that the oft-cited 5-10-ms echo threshold for equal-amplitude lead-lag transients is a significant underestimate of the delay at which "echoes" actually become discretely perceptible, or problematic, in most environments (cf. Hafter et al. 2001).

In a separate domain, the contribution of visual information to the $\mathrm{PE}$ is an area ripe for investigation, with recent indications that visual information readily influences the perceptibility of the lead or lag (e.g., Bishop et al. 2011, 2012; if the lead or lag is visually reinforced, the lag is perceived less or more often). Parametric studies of the influence of visual information on sound localization and the $\mathrm{PE}$ in particular will improve our understanding of how the PE operates ecologically. While some species localize 
sound in the absence of visual cues, such that purely auditory stimuli may be ecological, humans (and many models used for translational reasons) generally do not. Experiments that bias auditory versus visual information (cf. Bishop et al. 2011), for example, will work to establish the relative dominance of each modality for localization in reverberant settings.

Relationships Among Psychophysical Measures of the Precedence Effect. While fusion, localization dominance, and discrimination suppression are now generally well-defined, their functional interrelationships remain unclear. This is not an entirely semantic problem. The fusion aspect of the PE determines the number of images perceived. This aspect of the phenomenon, at least, can be (and has been) measured independent of localization dominance or discrimination suppression. It appears that under some circumstances, fusion and localization dominance can change independently of one another. Summing localization (strong fusion without localization dominance) is one example, but some data suggest similar dissociation at long (10-20 ms) lead-lag delays. Further investigation of this matter will work to elucidate which aspects of the PE may be attributable to nonspatial mechanisms and which are explicitly spatial in nature.

Physiological Mechanisms That Give Rise to the Precedence Effect. While the PE has been studied at nearly every site in the auditory system, the manner in which a PE stimulus is processed by the intact auditory system or even brainstem remains unknown. In particular, although it seems clear that important correlates of the PE emerge at the level of the IC and that the DNLL is positioned to contribute to spatially dependent lag suppression (e.g., Burger and Pollak 2001; see Tollin et al. 2004; Pecka et al. 2007; Xia et al. 2010), details of this circuit remain obscure. Pharmacological, genetic, or optogenetic studies that provide for focused, reproducible, and most ideally reversible inactivation of one or both DNLLs in awake, behaving animals, for example, would enable study of the functional contributions of DNLL's ipsilateral projections to IC and contralateral projections to IC and the opposite DNLL to physiological and psychophysical aspects of the PE.

Contributions to the PE of structures beyond of IC are poorly understood. Animal data suggest a more robust PE in the auditory cortex than in the auditory midbrain, in that the cortical response to lag does not recover until well after the midbrain response has recovered. Is this difference in time course due to additional synaptic inhibition at the level of the cortex? What might the auditory thalamus contribute to PE processing in the cortex, or the colliculus? Is the cortico-collicular system (efferent projections from the cortex to the midbrain, see Winer 2006) involved in PE processing, perhaps for long-duration stimuli or under conditions of repeated stimulation? While physiological effects of repeated stimulation have been studied very little, some electrophysiological data from humans suggest that relatively "late" cortical events may contribute to the phenomenon of buildup. A cortical cooling experiment in an animal model (for reversible deactivation of the cortex; e.g., Nakamoto et al. 2008) paired with presentation of single and multiple lead-lag stimuli during recording from the IC would likely yield significant new insight on the role of the cortex in the PE.

Clinical Significance of the Precedence Effect. It is not clear to what degree or in what manner hearing loss affects the PE. Understanding limitations on or changes to the PE in hearing-impaired populations could contribute significantly to an improved understanding of notorious limitations on patient outcomes (e.g., persistently poor communication in acoustically complex social settings). Systematic investigations of the $\mathrm{PE}$ in hearing-impaired indivduals will elucidate possible links between hearing loss and changes in the PE specifically. It will be critical, in designing such investigations, to account for subject factors such as type of hearing loss, duration of hearing loss, and age. Finally, additional studies in users of cochlear implants will further establish the extent to which aspects of the PE are preserved in electric hearing, which may in turn provide further mechanistic insight (since cochlear implants bypass the inner ear).

\section{On the Ecological Utility of Precedence Effect}

By published accounts, the PE appears to be nearly as ubiquitous as audition itself. Precedence-like phenomena (prime salience of early-arriving spatial information) have been reported in nearly every organism examined, from parasitic flies to amphibians to birds to rodents to felines to canines to humans (but see data on echolocating bats, Schuchmann et al. 2006, and echolocating dolphins, Zaslavski 2008). The premises and interpretations of these many studies have often focused on the utility of PEs for echo suppression, that is, avoidance of the perceptual effects of echoes that might otherwise confound sound localization in real environments. This view, in turn, holds that the PE facilitates (depending on the organism) communication, environmental awareness, prey capture, and/or predator avoidance in natural environments. Though we, too, have discussed the PE largely in terms of sound localization in reverberant environments, we feel that this view deserves more scrutiny.

A major caveat for consideration (also suggested by Litovsky et al. 1999) concerns the actual prevalence of reflected sound in the natural world. The evolution of a system specifically for echo suppression would imply that reflected sound is a basic challenge that auditory systems have been obliged to overcome-not just once 
but likely multiple times, consistent with the multiple evolutionary lineages of audition and sound localization specifically (e.g., Schnupp and Carr 2009). In man-made environments abounding with parallel surfaces, the amplitudes of discrete signal reflections can be considerable but are still much less than the amplitudes of incident signals (e.g., Fig. 1). In natural environments, parallel or even planar surfaces, excepting the ground, and perhaps underground burrows or caves, are rare; thus, high-amplitude, highly directional reflections are also rare (although diffuse, low-intensity reflections, e.g., see bottom panel of Fig. 1, still carry some information about the spatial extent of the environment, and may even reinforce the direct signal). Thus, returning to the laboratory, lead and lag signals with equal intensity, or even only moderately lower intensity in the lag, would seem to substantially exaggerate the ecological problem of reflected sound, especially for species that $d o$ operate in relatively anechoic environments, e.g., owls in flight.

Zurek (1980) suggested an alternative view-that the PE is useful for "the avoidance of interaural ambiguities" (1980). While such ambiguities can arise as a result of reflected sound, interaural ambiguities can also arise as a result of competing signals (e.g., multiple communication signals from conspecifics, Bosch and Marquez 2002; Lee et al. 2009), as a result of interaural decorrelation (e.g., diffuse reverberation, rather than spatially coherent reflections), or as a result of interaural phase ambiguities that arise for highfrequency signals, for which the signal wavelength is less than the width of the head. In each of these cases, a mechanism that emphasizes the leading signal offers to disambiguate the spatial acoustic cues so that a meaningful location can be ascribed to the auditory event, providing a basis for behavioral action. The question of what the PE is good for is of course separate from the empirical question of how the laboratory PE works. Indeed, the synthetic lead-lag stimulus used in so many investigations has proven a utile stimulus for measuring basic temporal and spatial aspects of auditory perception, for interrogating the function of auditory brainstem circuits, and for evaluating the predictive power of models of auditory processing. Nonetheless, a more ecological understanding of the $\mathrm{PE}$ as a mechanism for the preservation of accurate sound localization in reverberant environments or other adaptive purposes will ultimately require more ecological approaches to its study.

\section{ACKNOWLEDGMENTS}

The authors would like to thank Editor-in-Chief Paul Manis, Associate Editor Ruth Anne Eatock and two anonymous reviewers for comments on the manuscript. This work was supported by the National Institute on Deafness and Other Communication Disorders (NIDCD) grant R01-DC011555 (DJT), R01-DC011548 (GCS), and F32-DC013927 (ADB).

\section{REFERENCES}

Adams JC, Mugnaini E (1984) Dorsal nucleus of the lateral lemniscus: a nucleus of GABAergic projection neurons. Brain Res Bull 13:585-590

Agaeva MY, Al'tman YA (2008) Echo thresholds measured in the vertical and horizontal planes. Hum Physiol 34:678-684

Agrawal SS (2008) Spatial hearing abilities in adults with bilateral cochlear implants. Doctoral dissertation. University of Wisconsin - Madison

Akeroyd MA, Guy FH (2011) The effect of hearing impairment on localization dominance for single-word stimuli. J Acoust Soc Am 130:312

Alain C, Arnott S, Picton T (2001) Bottom-up and top-down influences on auditory scene analysis: evidence from eventrelated brain potentials. J Exp Psychol: Hum Percept Perform 27:1072-89

ANDREASEN NC (1997) The role of the thalamus in schizophrenia. Can J Psychiatry 42:27-33

Backer KC, Hill KT, Shahin AJ, Miller LM (2010) Neural time course of echo suppression in humans. J Neurosci 30:1905-1913

Baxter CS, Nelson BS, Takahashi TT (2013) The role of envelope shape in the localization of multiple sound sources and echoes in the barn owl. J Neurophysiol 109:924-31

Bernstein LR, Trahiotis C (2002) Enhancing sensitivity to interaural delays at high frequencies by using 'transposed stimuli'. J Acoust Soc Am 112:1026-1036

Bianchi F, Verhulst S, Dau T (2013) Experimental evidence for a cochlear source of the precedence effect. J Assoc Res Otolaryngol 14:767-779

Bilsen FA, RAATgever J (1973) Spectral dominance in binaural lateralization. Acustica 28:131-132

Bishop CW, London S, Miller LM (2011) Visual influences on echo suppression. Curr Biol 21:221-225

Bishop CW, London S, Miller LM (2012) Neural time course of visually enhanced echo suppression. J Neurophysiol 108:18691883

BlaUert J (1997) Spatial hearing: the psychophysics of human sound localization, revised edition. The MIT Press, Cambridge

Blauert J, Cobben W (1978) Some consideration of binaural crosscorrelation analysis. Acta Acust united Acust 39(2):96-104

Blauert J, Divenyi PL (1988) Spectral selectivity in binaural contralateral inhibition. Acta Acust united Acust 66(5):267-274

Bosch, Marquez (2002) Female preference function related to precedence effect in an amphibian anuran (Alytes cisternasii): tests with non-overlapping calls. Behav Ecol 13:149-153

BrAASCH J (2013) A precedence effect model to simulate localization dominance using an adaptive, stimulus parameter-based inhibition process. J Acoust Soc Am 134:420-435

Braasch J, Blauert J (2003) The precedence effect for noise bursts of different bandwidths. II. Comparison of model algorithms. Acoust Sci Technol 24:5

Braasch J, Blauert J, Djelani T (2003) The precedence effect for noise bursts of different bandwidths. I. Psychoacoustical data. Acoust Sci Tech 24:233-241

Brandewie E, Zahorik P (2010) Prior listening in rooms improves speech intelligibility. J Acoust Soc Am 128:291-299

Breebaart J, van de Par S, Kohlrausch A (2001A) Binaural processing model based on contralateral inhibition. I. Model structure. J Acoust Soc Am 110:1074-1088

Breebaart J, van de Par S, Kohlrausch A (2001в) Binaural processing model based on contralateral inhibition. I. Dependence on spectral parameters. J Acoust Soc Am 110:1089-1104

Brown AD, Stecker GC (2010) Temporal weighting of interaural time and level differences in high-rate click trains. J Acoust Soc Am 128:332-341 
Brown AD, Stecker GC (2011) Temporal weighting of interaural time and level differences in high-rate click trains. II: the effect of binaurally synchronous temporal jitter. J Acoust Soc Am 129:293-300

Brown AD, Stecker GC (2012) The precedence effect in sound localization: distinct roles for interaural time and level differences suggested by behavioral, modeling and acoustic data. Assoc Res Otolaryngol Abstr 35:648

Brown AD, Stecker GC (2013) The precedence effect in sound localization: fusion and lateralization measures for pairs and trains of clicks lateralized by interaural time and level differences. J Acoust Soc Am 133:2883-2898

Brown AD, Jones HG, Kan AH, Stecker GC, Goupell MJ, and Litovsky RY (2013) The precedence effect: insights from electric hearing. Conf. on Implantable Auditory Prostheses, abstract M10

Brugge JF, Anderson DJ, Aitkin LM (1970) Responses of neurons in the dorsal nucleus of the lateral lemniscus of cat to binaural tonal stimulation. J Neurophysiol 33:441-458

Brughera A, Dunai L, Hartmann WM (2013) Human interaural time difference thresholds for sine tones: the high frequency limit. J Acoust Soc Am 133:2839

Burger RM, Pollak GD (2001) Reversible inactivation of the dorsal nucleus of the lateral lemniscus reveals its role in the processing of multiple sound sources in the inferior colliculus of bats. J Neurosci 21:4830-4843

CARNey LH (1993) A model for the responses of low-frequency auditory-nerve fibers in cat. J Acoust Soc Am 93:401

CARNeY LH, YIN TCT (1989) Responses of low-frequency cells in the inferior colliculus to interaural time differences of clicks: excitatory and inhibitory components. J Neurophysiol 62:144161

CLIFTON RK (1987) Breakdown of echo suppression of the precedence effect. J Acoust Soc Am 82:1834-1835

Chifton RK, Freyman RL (1989) Effect of click rate and delay on breakdown of the precedence effect. Percept Psychophys 46:139-145

Clifton RK, Morrongiello BA, Dowd JM (1984) A developmental look at an auditory illusion: the precedence effect. Dev Psychol $17: 519-536$

Chifton RK, Freyman RL, Litovsky RY, McCall D (1994) Listeners' expectations about echoes can raise or lower echo threshold. J Acoust Soc Am 95:1525-1533

Colburn HS (1973) Theory of binaural interaction based on auditory-nerve data. I. General strategy and preliminary results on interaural discrimination. J Acoust Soc Am 54:1458-1470

CRANFORd JL (1982) Localization of paired sound sources in cats: effects of variable arrival times. J Acoust Soc Am 72:1309-11

Cranford JL, Boose M, Moore CA (1990) Tests of the precedence effect in sound localization reveal abnormalities in multiple sclerosis. Ear Hear 11:282-288

Cranford JL, Andres MA, Piatz KK, Reissig KL (1993) Influences of age and hearing loss on the precedence effect in sound localiztion. J Speech Lang Hear Res 36:437

Cremer L (1948) Geometrische raumakustik. Die Wissenschaftlichen Grundlagen der Raumakustik (S. Hertzel, Stuttgart) vol. 1, p. 126

Damaschke J, Riedel H, Kollmeier B (2005) Neural correlates of the precedence effect in auditory evoked potentials. Hear Res 205:157-171

Dent ML, Dooling RJ (2003A) Investigations of the precedence effect in budgerigars: effects of stimulus type, intensity, duration, and location. J Acoust Soc Am 113:2146-2158

Dent ML, Dooling RJ (2003B) Investigations of the precedence effect in budgerigars: the perceived location of auditory images. J Acoust Soc Am 113:2159-2169

Dent ML, Tollin DJ, YIN TCT (2009) Influence of sound source location on the behavior and physiology of the precedence effect in cats. J Neurophysiol 102:724-734
Devore S, Delgutte B (2010) Effects of reverberation on the directional sensitivity of auditory neurons across the tonotopic axis: influences of interaural time and level differences. J Neurosci 30:7826-7837

Devore S, Ihlefeld A, Hancock K, Shinn-Cunningham BG, Delgutte B (2009) Accurate sound localization in reverberant environments is mediated by robust encoding of spatial cues in the auditory midbrain. Neuron 62:123-134

Dietz M, Ewert S, Hohmann V (2011) Auditory model based direction estimation of concurrent speakers from binaural signals. Speech Comm 53:592-605

Dietz M, Marquardt T, Salminen NH, McAlpine D (2013) Emphasis of spatial cues in the temporal fine structure during the rising segments of amplitude-modulated sounds. Proc Nat Acad Sci 110:15151-15156

Dimitrijevic A, Stapells DR (2006) Human electrophysiological examination of the buildup of the precedence effect. Neuroreport 17:1133-1137

Dizon RM, Colburn HS (2006) The influence of spectral, temporal, and interaural stimulus variations on the precedence effect. J Acoust Soc Am 119:2947-2964

Dizon RM, Litovsky RY (2004) Localization dominance in the median-sagittal plane: effect of stimulus duration. J Acoust Soc Am 115:3142-3155

Djelani T, Blauert J (2001) Investigations into the build-up and breakdown of the precedence effect. Acta Acustica 87:253-261

Donovan JM, Nelson BS, Takahashi TT (2012) The contributions of onset and offset echo delays to auditory spatial perception in human listeners. J Acoust Soc Am 132:3912-3924

Ebata M, Sone T, Nimura T (1968) On the perception of direction of echo. J Acoust Soc Am 44:542-547

Faller C, Merimaa J (2004) Source localization in complex listening situations: selection of binaural cues based on interaural coherence. J Acoust Soc Am 116:3075-3089

FAY RD (1936) A method for obtaining natural directional effects in a public address system. J Acoust Soc Am 7:239

Fitzpatrick DC, Kuwada S, Batra R, Trahiotis C (1995) Neural responses to simple simulated echoes in the auditory brain stem of the unanesthetized rabbit. J Neurophysiol 74:2469-2485

Fitzpatrick DC, Kuwada S, Kim DO, Parham K, Batra R (1999) Responses of neurons to click-pairs as simulated echoes: auditory nerve to auditory cortex. J Acoust Soc Am 106:3460-72

Franssen NV (1960) Some considerations on the mechanism of directional hearing. Doctoral Disseration. Technische Hogeschool, Delft, The Netherlands

Freyman RL, KeEn R (2006) Constructing and disrupting listeners' models of auditory space. J Acoust Soc Am 120:3957-3965

Freyman RL, Clifton RK, Litovsky RY (1991) Dynamic processes in the precedence effect. J Acoust Soc Am 90:874-884

Freyman RL, Zurek PM, Balakrishnan U, Chiang YC (1997) Onset dominance in lateralization. J Acoust Soc Am 101:1649-1659

Gai Y, Ruhland JL, Yin TC, Tollin DJ (2013) Behavioral and modeling studies of sound localization in cats: effects of stimulus level and duration. J Neurophysiol 110:607-620

GARDNER MB (1968) Historical background of the Haas and/or precedence effect. J Acoust Soc Am 43:1244-1248

Gaskell H (1983) The precedence effect. Hear Res 12:277-303

Gonzalez-Hernandez T, Mantolan-Sarmiento B, GonZalez-Gonzalez B, Perez-Gonzalez H (1996) Sources of GABAergic input to the inferior colliculus of the rat. J Comp Neurol 372:309-326

Goupell MJ, LABACK B, MajDAK P (2009) Enhancing sensitivity to interaural time differences at high modulation rates by introducing temporal jitter. J Acoust Soc Am 126:2511-2521

Goupell MJ, Yu G, Litovsky RY (2012) The effect on an additional reflection in a precedence effect experiment. J Acoust Soc Am 131:2958-2967 
Goverts ST, Houtgast T, van Beek HHM (2002) The precedence effect for lateralization for the mild sensory neural hearing impaired. Hear Res 163:82-92Grantham WD (1996) Left-right asymmetry in the buildup of echo suppression in normal-hearing adults. J Acoust Soc Am 99:1118-1123

Grantham WD, Ashmead DH, Ricketts TA, Haynes DS, Labadie RF (2008) Interaural time and level difference thresholds for acoustically presented signals in post-lingually deafened adults fitted with bilateral cochlear implants using CIS+ processing. Ear Hear 29:33-44

HaAs H (1949) The influence of a single echo on the audibility of speech. J Audiol Eng Soc 20:145-159

HaAs H (1951) On the influence of a single echo on the intelligibility of speech. Acustica 1:48-58

HAFTER ER (1997) Binaural adaptation and the effectiveness of a stimulus beyond its onset. In: H Gilkey and TR Anderson (Eds.) Binaural and spatial hearing in real and virtual environments, (Maywah, NJ: Lawrence Erlbaum Associates) pp. 211-232

Hafter ER, Buell TN (1990) Restarting the adapted binaural system. J Acoust Soc Am 88:806-812

HAFter ER, Dye RH (1983) Detection of interaural differences of time in trains of high-frequency clicks as a function of interclick interval and number. J Acoust Soc Am 73:644-651

Hafter ER, Dye RH, Wenzel E (1983) Detection of interaural differences of intensity in trains of high-frequency clicks as a function of interclick interval and number. J Acoust Soc Am 73:1708-1713

Hafter ER, Buell TN, Richards VM (1988) Onset-coding in lateralization: its form, site, and function. In: Edelman GM, Gall WE, Cowan WM (eds) Auditory function: neurobiological bases of hearing. Wiley, New York, pp 647-676

Hafter ER, Dye RH, Wenzel E, Knecht K (1990) The combination of interaural time and intensity in the lateralization of highfrequency complex signals. J Acoust Soc Am 87:1702-1708

Hafter ER, Valenzuela MN, Stecker GC, Miele JA, and Crum PaC (2001) Informational dominance in the auditory scence. In: Physiological and psychophysical bases of auditory function. Breebaart, Houtsma, Kohlrausch, Prijs, and Schoonhoven (Eds.) (Maastricht NL: Shaker) pp. 208-214

HALL WM (1936) A method for maintaining in a public address system the illusion that the sound comes from the speaker's mouth. J Acoust Soc Am 7:239

Hartung K, Trahiotis C (2001) Peripheral auditory processing and investigations of the 'precedence effect' which utilize successive transient stimuli. J Acoust Soc Am 110:1505-1513

Heller LM, Trahiotis C (1996) Extents of laterality and binaural interference effects. J Acoust Soc Am 99:3632-3637

Hutson KA, Glendenning KK, Masterton RB (1991) Acoustic chiasm IV: eight midbrain decussations of the auditory system in the cat. J Comp Neurol 312:105-131

Ihlefeld A, Shinn-Cunningham BG (2011) Effects of source spectrum on sound localization in an everyday reverberant room. J Acoust Soc Am 130:324-333

Keen R, Freyman RL (2009) Release and re-buildup of listeners' models of auditory space. J Acoust Soc Am 125:3243-3252

Keller CH, Takahashi TT (1996) Responses to simulated echoes by neurons in the barn owl's auditory space map. J Comp Physiol A 178:499-512

KeLly JB (1974) Localization of paired sound sources in the rat: small time differences. J Acoust Soc Am 55:1277-1284

Kelly JB, Buckthought A, Kidd SA (1998) Monaural and binaural properties of single neurons in the rat's dorsal nucleus of the lateral lemniscus. Hear Res 122:25-40

Kerber S, Seeber BU (2013) Localization in reverberation with cochlear implants. J Assoc Res Otolaryngol 14:379-392
Kiang NYS, Watanabe T, Thomas EC, Clark LF (1965) Discharge patterns of single fibers in the cat's auditory nerve. MIT Press, Cambridge, Research Monograph 35

Krumbholz K, Nobbe A (2002) Buildup and breakdown on echo suppression for stimuli presented over headphones-the effects of interaural time and level differences. J Acoust Soc Am 112:654-663

Lee N, Elias DO, Mason AC (2009) A precedence effect resolves phantom sound source illusions in the parasitoid fly Ornia ochracea. Proc Nat Acad Sci 106:6357-6362

Liebenthal E, Pratt H (1999) Human auditory cortex electrophysiological correlates of the precedence effect: binaural echo lateralization suppression. J Acoust Soc Am 106:291-303

LiNDEMANN W (1986A) Extension of a binaural cross-correlation model by contralateral inhibition. I. Simulation of lateralization for stationary signals. J Acoust Soc Am 80:1608-1622

Lindemann W (1986B) Extension of a binaural cross-correlation model by contralateral inhibition. II. The law of the first wavefront. J Acoust Soc Am 80:1623-1630

Lister JJ, Roberts RA (2005) Effects of age and hearing loss on gap detection and the precedence effect: narrow-band stimuli. J Speech Lang Hear Res 48:482-493

Litovsky RY (1997) Developmental changes in the precedence effect: estimates of minimal audible angle. J Acoust Soc Am 102:1739-1745

Litovsky RY, DeLGUtTe B (2002) Neural correlates of the precedence effect in the inferior colliculus: effect of localization cues. J Neurophysiol 87:976-994

Litovsky RY, GoDar SP (2010) Difference in precedence effect between children and adults signifies development of sound localization abilities in complex listening tasks. J Acoust Soc Am 128:1979-1991

Litovsky RY, Shinn-Cunningham BG (2001) Investigation of the relationship among three common measures of precedence: fusion, localization dominance, and discrimination suppression. J Acoust Soc Am 109:346-358

Litovsky RY, YIN TCT (1998A) Physiological studies of the precedence effect in the inferior colliculus of the cat. I. Correlates of psychophysics. J Neurophysiol 80:1285-301

Litovsky RY, YIN TCT (1998B) Physiological studies of the precedence effect in the inferior colliculus of the cat. II. Neural mechanisms. J Neurophysiol 80:1302-16

Litovsky RY, Colburn HS, Yost WA, Guzman SJ (1999) The precedence effect. J Acoust Soc Am 106:1633-1654

Litovsky RY, Fligor BJ, Tramo MJ (2002) Functional role of the human inferior colliculus in binaural hearing. Hear Res 165:177-188

Litovsky RY, Johnstone PM, Godar S (2006) Benefits of bilateral cochlear implants and/or hearing aids in children. Int J Audiol 45:78-91

Litovsky RY, Jones GL, Agrawal S, van Hoesel RJM (2010) Effect of age at onset of deafness on binaural sensitivity in electric hearing in humans. J Acoust Soc Am 127:400-414

LuCE RD (1986) Response times: their role in inferring elementary mental organization. Oxford University Press, Oxford

Macpherson EA, Wagner ML (2008) Temporal weighting of cues for vertical-plane sound localization. Assoc Res Otolaryngol Abstr 31:882

Markovitz NS, Pollak GD (1994) Binaural processing in the dorsal nucleus of the lateral lemniscus. Hear Res 73:121-140

McCall DD, Freyman RL, Clifton RK (1998) Sudden changes in spectrum of an echo cause a breakdown of the precedence effect. Percept Psychophys 60:593-601

Meddis R (1986) Simulation of mechanical to neural transduction in the auditory receptor. J Acoust Soc Am 79:702-711

Mickey BJ, DALAck GW (2005) Auditory gating in schizophrenia: a pilot study of the precedence effect. Schizophr Res 73:327-331 
Mickey BJ, Middlebrooks JC (2001) Responses of auditory cortical neurons to pairs of sounds: correlates of fusion and localization. J Neurophysiol 86:1333-50

Mickey BJ, Middlebrooks JC (2005) Sensitivity of auditory cortical neurons to the locations of leading and lagging sounds. J Neurophysiol 94:979-989

Miller SD, Litovksy RY, Kluender KR (2009) Predicting echo thresholds from speech onset characteristics. J Acoust Soc Am 125:EL135

Moore JM, Tollin DJ, Yin TC (2008) Can measures of sound localization acuity be related to the precision of absolute location estimates? Hear Res 238:94-109

Nakamoto KT, Jones SJ, Palmer AR (2008) Descending projections from auditory cortex modulate sensitivity in the midbrain to cues for spatial position. J Neurophysiol 99:2347-2356

Nelson BS, Takahashi TT (2008) Independence of echo-threshold and echo-delay in the barn owl. PLoS ONE 3(10):e3598

Nelson BS, Takahashi TT (2010) Spatial hearing in echoic environments: the role of the envelope in owls. Neuron 67:643-55

Parham K, Zhao HB, Kim DO (1996) Responses of auditory nerve fibers of the unanesthetized decerebrate cat to click pairs as simulated echoes. J Neurophysiol 76:17-29

Parham K, Zhao HB, Ye Y, Kim DO (1998) Responses of anteroventral cochlear nucleus neurons of the unanesthetized decerebrate cat to click pairs as simulated echoes. Hear Res 125:131-46

Pecka M, Zahn TP, Saunier-Rebori B, Siveke I, Wiegrebe L, Klug A, Pollak GD, Grothe B (2007) Inhibiting the inhibition: a neuronal network for sound localization in reverberant environments. J Neurosci 267:1782-1790

Populin LC (2006) Monkey sound localization: head-restrained vs head-unrestrained orienting. J Neurosci 26:9820-9832

RAKERD B, HARTMANN WM (1985) Localization of sound in rooms, II: the effects of a single reflecting surface. J Acoust Soc Am 78:524533

Rakerd B, Hartmann WM, Hsu J (2000) Echo suppression in the horizontal and median sagittal planes. J Acoust Soc Am 107:1061-1064

Reale RA, Brugge JF (2000) Directional sensitivity of neurons in the primary auditory (AI) cortex of the cat to successive sounds ordered in time and space. J Neurophysiol 84:435-50

Roberts RA, Lister JJ (2004) Effects of age and hearing loss on gap detection and the precedence effect: broadband stimuli. J Speech Lang Hear Res 47:965-978

Roberts RA, Besing J, KoehnKe J (2002) Effects of hearing loss on echo thresholds. Ear Hear 23:349-357

Roberts R, Koehnke J, Besing J (2003) Effects of noise and reverberation on the precedence effect in listeners with normal hearing and hearing loss. Am J Audiol 12:96-105

SABERI K (1996) Observer weighting of interaural delays in filtered impulses. Percept Psychophys 58:1037-1046

Saberi K, Antonio JV (2003) Precedence-effect thresholds for a population of untrained listeners as a function of stimulus intensity and interclick interval. J Acoust Soc Am 114:420-429

SAberi K, Perrot DR (1995) Lateralization of click-trains with opposing onset and ongoing interaural delays. Acustica 81:272275

Saberi K, Antonio JV, Petrosyan A (2004) A population study of the precedence effect. Hear Res 191:1-13

Sanders LD, Joh AS, Freyman RL, Keen R (2008) One sound or two? Object-related negativity indexes echo suppression. Percept Psychophys 70:1558-1570

Sanders LD, Zobel B, Keen R, Freyman RL (2011) Manipulations of listeners' echo perception are reflected in event-related potentials. J Acoust Soc Am 129:301-309
SCHNUPP JWH, CARR CE (2009) On hearing with more than one ear: lessons from evolution. Nat Neurosci 12:692-697

Schuchmann M, Hübner M, Wiegrebe L (2006) The absence of spatial echo suppression in the echolocating bats Megaderma lyra and Phyllostomus discolor. J Exp Biol 209:152-157

SeEber BU, Hafter ER (2008) Parameters affecting the precedenceeffect with cochlear implants. J Acoust Soc Am 123:3055

SEeBER BU, HAFter ER (2011) Failure of the precedence effect with a noise band vocoder. J Acoust Soc Am 129:1509-1521

Shinn-Cunningham BG, Zurek PM, Durlach NI (1993) Adjustment and discrimination measurements of the precedence effect. J Acoust Soc Am 98:164-171

Shinn-Cunningham GC, Zurek PM, Durlach NI, Clifton RK (1995) Cross frequency interactions in the precedene effect. J Acoust Soc Am 98:164-171

Shneiderman A, Oliver DL, Henkel C (1988) Connections of the dorsal nucleus of the lateral lemniscus: an inhibitory parallel pathway in the ascending auditory system? J Comp Neurol 276:188-208

Shneiderman A, Stanforth DA, Henkel CK, Saint Marie RL (1999) Input-output relationships of the dorsal nucleus of the lateral lemniscus: possible substrate for the processing of dynamic spatial cues. J Comp Neurol 410:265-276

SNow WB (1936) Sound reproducing system. U.S Patent 2,137,032.

Song P, WAng N, Wang H, XIE Y, Li H (2011) Pentobarbital anesthesia alters neural responses in the precedence effect. Neurosci Lett 498:72-77

Spierer L, Bourquin NM-P, Tardif E, Murray MM, Clarke S (2009) Right hemispheric dominance for echo suppression. Neuropsychologia 47:465-472

SPITZER MW, TAKAHASHI TT (2006) Sound localization by barn owls in a simulated echoic environment. J Neurophysiol 95:3571-3584

Spitzer MW, Bala AD, TAKahashi TT (2003) Auditory spatial discrimination by barn owls in simulated echoic conditions. J Acoust Soc Am 113:1631-45

Sittzer MW, BALA AD, TAKAHASHI TT (2004) A neuronal correlate of the precedence effect is associated with spatial selectivity in the barn owl's auditory midbrain. J Neurophysiol 92:2051-2070

STECKER GC, BiBEe JM (2014) Nonuniform temporal weighting of interaural time differences in $500 \mathrm{~Hz}$ tones. J Acoust Soc Am 135:3541-3547

SteCKER GC, Brown AD (2010) Temporal weighting of binaural cues revealed by detection of dynamic interaural differences in highrate Gabor click trains. J Acoust Soc Am 127:3092-3103

Stecker GC, Brown AD (2012) Onset- and offset-specific effects in interaural level difference discrimination. J Acoust Soc Am 132:1573-1580

Stecker GC, Hafter ER (2002) Temporal weighting in sound localization. J Acoust Soc Am 112:1046-1057

Stecker GC, Hafter ER (2009) A recency effect in sound localization? J Acoust Soc Am 125:3914-3924

Stecker GC, Ostreicher JO, Brown AD (2013) Temporal weighting functions for interaural time and level differences: III. Measurements using open-loop lateralization tasks. J Acoust Soc Am 134:1242-1252

Stellmack MA, Dye RH, Guzman SJ (1999) Observer weighting of binaural information in source and echo clicks. J Acoust Soc Am 105:377-387

Stern RM, Zeilberg AS, Trahiotis C (1988) Lateralization of complex binaural stimuli: a weighted-image model. J Acoust Soc Am 84:952-964

Stevens SS, Newman EB (1936) The localization of actual sources of sound. Am J Psychol 48:297-306

Takanen M, Sanatala O, Pulkki V (2013) Visualization of functional count-comparison- based binaural auditory model output. Hear Res. doi:10.1016/j.heares.2013.10.004 
Tollin DJ (1998) Computational model of the lateralization of clicks and their echoes. In: Proceedings of the NATO Advanced Study Institute on Computational Hearing, S. Greenberg and M. Slaney (Eds.), (Amsterdam: IOS Press) pp. 77-82.

Tollin DJ, Henning GB (1998) Some aspects of the lateralization of echoed sound in man. I. The classical interaural-delay based precedence effect. J Acoust Soc Am 104:3030-3038

Tollin DJ, Henning GB (1999) Some aspects of the lateralization of echoed sound in man. II. The role of the stimulus spectrum. J Acoust Soc Am 105:838-849

Tollin DJ, YIN TCT (2003) Psychophysical investigation of an auditory spatial illusion in cats: the precedence effect. J Neurophysiol 90:2149-2162

Tollin DJ, Populin LC, YIN TCT (2004) Neural correlates of the precedence effect in the inferior colliculus of behaving cats. J Neurophysiol 92:3286-3297

Tollin DJ, Ruhland JL, YIN TCT (2009) The vestibulo-auricular reflex. J Neurophysiol 101:1258-1266

Tollin DJ, McClaine L, YIN TCT (2010) Short-latency, goal-directed movements of the pinnae to sounds that produce auditory spatial illusions. J Neurophysiol 103:446-457

Tollin DJ, Ruhland JL, Yin TCT (2013) The role of spectral composition of sounds on the localization of sound sources by cats. J Neurophysiol 109:1658-1668

Tolnai S, Litovsky RY, KING AJ (2014) The precedence effect and its buildup and breakdown in ferrets and humans. J Acoust Soc Am 135:1406-1418

van Hoesel RJM (2007) Sensitivity to binaural timing in bilateral cochlear implant users. J Acoust Soc Am 121:2192-2206

Wallach H, Newman EB, Rosenzweig R (1949) The precedence effect in sound localization. Am J Psychiatr 62:315-336

WARNCKE H (1941) The fundamentals of room-related stereophonic reproduction in sound films. Akust Zh 6:174-188

WICKESBERG RE (1996) Rapid inhibition in the cochlear nuclear complex of the chinchilla. J Acoust Soc Am 100:1691-1702

Wickesberg RE, Oertel D (1990) Delayed, frequency-specific inhibition in the cochlear nuclei of mice: a mechanism for monaural echo suppression. J Neurosci 10:1762-1768

Wightman FL, Kistler DJ (1992) The dominant role of lowfrequency interaural time differences in sound localization. J Acoust Soc Am 91:1648-1661
Winer JA (2006) Decoding the auditory corticofugal systems. Hear Res 212:1-8

WoLF S (1991) Untersuchungen zur Lokalisation von Schallquellen in geschlossenen Räumen, Dissertation, Ruhr-Universitaät Bochum

Wolf M, Schuchmann M, Wiegrebe L (2010) Localization dominance and the effect of frequency in the Mongolian Gerbil, Meriones unguiculatus. J Comp Physiol A 196:463-470

Xia J, Shinn-Cunningham BG (2011) Isolating mechnisms that influence measures of the precedence effect: theoretical predictions and behavioral tests. J Acoust Soc Am 130:866-882

Xia J, Brughera A, Colburn HS, Shinn-Cunningham BG (2010) Physiological and psychophysical modeling of the precedence effect. J Assoc Res Otolaryngol 11:495-513

YANG X, GRANTHAm DW (1997) Echo suppression and discrimination suppression aspects of the precedence effect. Percept Psychophys 59:1108-1117

YANG L, POLLAK GD (1994) Binaural inhibition in the dorsal nucleus of the lateral lemniscus of the mustache bat affects responses for multiple sounds. Aud Neurosci 1:1-17

YIN TCT (1994) Physiological correlates of the precedence effect and summing localization in the inferior colliculus of the cat. J Neurosci 14:5170-86

Yost WA (2007) Lead-lag precedence paradigm as a function of relative level and number of lag stimuli. Proceedings of the 19th International Congress on Acoustics. Madrid, Spain

Yost WA, Soderquist DR (1984) The precedence effect: revisited. J Acoust Soc Am 76:1377-1383

ZASLAVSKI GL (2008) Localization of brief sounds by a bottlenose dolphin. POMA: Acoustics 08 Paris:2803-2808.

Zilany MSA, Bruce IC, Nelson PC, Carney LH (2009) A phenomenological model of the synapse between the inner hair cell and auditory nerve: long-term adaptation with power-law dynamics. J Acoust Soc Am 126:2390-2412

ZuREK PM (1980) The precedence effect and its possible role in the avoidance of interaural ambiguities. J Acoust Soc Am 67:952-964

ZuREK PM (1987) The precedence effect. In: Yost WA, Gourevitch G (eds) Directional hearing. Springer-Verlag, New York, pp 85-105

Zwislocki J, Feldman RS (1956) Just noticeable differences in dichotic phase. J Acoust Soc Am 28:860-864 